\title{
Disentangling random thermal motion of particles and collective expansion of source from transverse momentum spectra in high energy collisions
}

\author{
Hua-Rong $\mathrm{Wei}^{a}, \mathrm{Fu}-\mathrm{Hu} \mathrm{Liu}^{a}\left[1\right.$, and Roy A. Lacey ${ }^{b}, 2$ \\ ${ }^{a}$ Institute of Theoretical Physics, Shanxi University, Taiyuan, Shanxi 030006, China \\ ${ }^{b}$ Departments of Chemistry $\&$ Physics, Stony Brook University, Stony Brook, NY 11794, USA
}

\begin{abstract}
In the framework of a multisource thermal model, we describe experimental results of the transverse momentum spectra of final-state light flavour particles produced in gold-gold $(\mathrm{Au}-\mathrm{Au})$, copper-copper $(\mathrm{Cu}-\mathrm{Cu})$, lead-lead $(\mathrm{Pb}-\mathrm{Pb})$, proton-lead $(p-\mathrm{Pb})$, and proton-proton $(p-p)$ collisions at various energies, measured by the PHENIX, STAR, ALICE, and CMS Collaborations, by using the Tsallisstandard (Tsallis form of Fermi-Dirac or Bose-Einstein), Tsallis, and two- or three-component standard distributions which can be in fact regarded as different types of "thermometers" or "thermometric scales" and "speedometers". A central parameter in the three distributions is the effective temperature which contains information on the kinetic freeze-out temperature of the emitting source and reflects the effects of random thermal motion of particles as well as collective expansion of the source. To disentangle both effects, we extract the kinetic freeze-out temperature from the intercept of the effective temperature $(T)$ curve as a function of particle's rest mass $\left(m_{0}\right)$ when plotting $T$ versus $m_{0}$, and the mean transverse flow velocity from the slope of the mean transverse momentum $\left(\left\langle p_{T}\right\rangle\right)$ curve as a function of mean moving mass $(\bar{m})$ when plotting $\left\langle p_{T}\right\rangle$ versus $\bar{m}$.
\end{abstract}

Keywords: Kinetic freeze-out temperature, Transverse flow velocity, Transverse momentum spectrum, Tsallis-standard distribution, Tsallis distribution, Standard distribution

PACS: 25.75.-q, 25.75.Ag, 25.75.Ld

\section{Introduction}

Comparing with fixed target experiments, the Relativistic Heavy Ion Collider (RHIC) in the USA and the Large Hadron Collider (LHC) in Switzerland attract more studies due to their exhibitions on the evolution process of interacting system in collisions at higher energies. The experiments at RHIC and LHC are more complex and difficult for some limited conditions, which render very limited measurable quantities. It is expected that more interacting information can be extracted from these limited measurable quantities. For example, by analyzing the transverse momentum spectra of final-state particles, one can obtain the kinetic freeze-out temperature of interacting systems (emission sources) and the transverse flow velocity of produced particles. These two quantities reflect the excited degree of emission sources and hydrodynamic expansion picture of interacting systems at the stage of kinetic freeze-out, when hadrons are no longer interactive and their momenta do not change [1].

In high energy collisions, one can use different distribution laws to describe the transverse momentum spectra of final-state particles. In the framework of a multisource thermal or statistical model, we can use the standard (Boltzmann, Fermi-Dirac, or Bose-Einstein) distribution [2], the multi-component standard distribution, the Tsallis distribution (statistics), the Tsallis-standard (Tsallis forms of the standard) distribution [3], the Erlang distribution, the multi-component Erlang distribution [4], the Lévy distribution $[5,6]$, the blast-wave function [7], the power law, and so forth, to describe the particle transverse momentum spectrum contributed by a given emission source. From the distributions mentioned above, one can directly extract the effective temperature, which is actually not the real temperature (kinetic freeze-out temperature) of the emission source. The real temperature of the emission source should reflect purely thermal motion of particles in the source, while the effective temperature extracted from the transverse momentum spectrum includes both thermal motion and flow effect of particles, and is greater than the

\footnotetext{
${ }^{1}$ E-mail: fuhuliu@163.com; fuhuliu@sxu.edu.cn

${ }^{2}$ E-mail: Roy.Lacey@Stonybrook.edu
} 
real temperature. Only if the effect of flow is excluded in the extraction of source temperature, can we obtain the kinetic freeze-out temperature.

In this paper, within the framework of the multisource thermal model [8-10], we use the Tsallisstandard distribution, Tsallis distribution, and two- or three-component standard distribution [3] to describe the transverse momentum spectra of final-state light flavour particles produced in gold-gold $(\mathrm{Au}-\mathrm{Au})$, copper-copper $(\mathrm{Cu}-\mathrm{Cu})$, lead-lead $(\mathrm{Pb}-\mathrm{Pb})$, proton-lead $(p-\mathrm{Pb})$, and proton-proton $(p$ - $p)$ collisions with different centrality intervals over a center-of-mass energy $\left(\sqrt{s_{N N}}\right)$ range from 0.2 to 7 TeV. Although we can obtain the analytical expressions for the mentioned distributions, the Monte Carlo method is used to see the statistical fluctuations in the process of calculation. The results from the Monte Carlo method are compared with the experimental data of the PHENIX [11], STAR [12], ALICE [13-18], and CMS Collaborations [19]. The kinetic freeze-out temperature of interacting system and transverse flow velocity of final-state particles are then extracted from the comparisons and analyses.

\section{The model and formulism}

In this paper, the transverse momentum spectra are described in the framework of the multisource thermal model [8-10], which assumes that many emission sources are formed in high energy collisions and are separated into a few groups resulting from different interacting mechanisms in the collisions as well as different event samples in experiment measurements. The sources in the same group have the same excitation degree and stay at a common local equilibrium state, which can be described by using different distribution laws. The final-state distribution is attributed to all sources in different groups, which results in a multi-temperature emission process if we use the standard distribution. This also means that the transverse momentum spectra can be described by a multi-component standard distribution which can be fitted by the Tsallis distribution. In fact, we can adopt one-component Tsallis-standard (T-S) distribution (Tsallis form of the Boltzmann, Fermi-Dirac, or Bose-Einstein distribution), one-component Tsallis distribution, and two- or three-component standard distribution (Boltzmann, Fermi-Dirac, or Bose-Einstein distribution), to describe the transverse momentum spectra of final-state light flavour particles.

According to refs. [3, 20], we use the uniform expressions of the Tsallis-standard distribution and Tsallis distribution which have more than one version [20-26], respectively. Based on the invariant particle momentum distribution, as well as the unit-density function of transverse momentum $\left(p_{T}\right)$ and rapidity $(y)$, the Tsallis-standard transverse momentum distribution and the Tsallis transverse momentum distribution are derived $[3,20]$. In the present work, the formalism of the Tsallis-standard transverse momentum distribution is adopted to be $[3,20]$

$f_{\mathrm{T}-\mathrm{S}}\left(p_{T}\right)=\frac{1}{N} \frac{d N}{d p_{T}}=C_{\mathrm{T}-\mathrm{S} 0} p_{T} \sqrt{p_{T}^{2}+m_{0}^{2}} \int_{y_{\min }}^{y_{\max }} \cosh y\left\{\left[1 \pm \frac{q_{\mathrm{T}-\mathrm{S}}-1}{T_{\mathrm{T}-\mathrm{S}}}\left(\sqrt{p_{T}^{2}+m_{0}^{2}} \cosh y-\mu\right)\right]^{ \pm \frac{1}{q_{\mathrm{T}-\mathrm{S}}-1}}+S\right\}^{-1} d y$,

where $C_{\mathrm{T}-\mathrm{S} 0}$ is the normalization constant $g V /(2 \pi)^{2}$ which results from $\int_{0}^{\infty} f_{\mathrm{T}-\mathrm{S}}\left(p_{T}\right) d p_{T}=1 ; g, V, N$, $\mu$, and $m_{0}$ are degeneracy factor, volume, particle number, chemical potential, and particle's rest mass, respectively; $y_{\min }$ is the minimum rapidity and $y_{\max }$ is the maximum rapidity; The + and - in the \pm sign are for $\sqrt{p_{T}^{2}+m_{0}^{2}} \cosh y>\mu$ and $\sqrt{p_{T}^{2}+m_{0}^{2}} \cosh y \leq \mu$, respectively; $S$ has values $0,+1$, and -1 , which denote the Boltzmann, Fermi-Dirac, and Bose-Einstein distributions, respectively; $T_{\mathrm{T}-\mathrm{S}}$ is the effective temperature of emission sources and $q_{\mathrm{T}-\mathrm{S}}$ is the entropy index or nonequilibrium degree factor.

In the calculation, since the effect of chemical potential can be ignored (i.e. $\mu=0$ ) in collisions at RHIC and LHC energies, we have $\sqrt{p_{T}^{2}+m_{0}^{2}} \cosh y>\mu$ and take + in the \pm sign. The final Tsallisstandard $p_{T}$ distribution is given by

$f_{\mathrm{T}-\mathrm{S}}\left(p_{T}\right)=\frac{1}{N} \frac{d N}{d p_{T}}=C_{\mathrm{T}-\mathrm{S} 0} p_{T} \sqrt{p_{T}^{2}+m_{0}^{2}} \int_{y_{\min }}^{y_{\max }} \cosh y\left\{\left[1+\frac{q_{\mathrm{T}-\mathrm{S}}-1}{T_{\mathrm{T}-\mathrm{S}}} \sqrt{p_{T}^{2}+m_{0}^{2}} \cosh y\right]^{\frac{1}{q_{\mathrm{T}-\mathrm{S}}-1}}+S\right\}^{-1} d y$.

Further, considering $S=0$ and the power index $q_{\mathrm{T}-\mathrm{S}} /\left(q_{\mathrm{T}-\mathrm{S}}-1\right)$ and other limitations, the Tsallis transverse momentum distribution is obtained and can be written as [20]

$$
f_{\mathrm{T}}\left(p_{T}\right)=\frac{1}{N} \frac{d N}{d p_{T}}=C_{\mathrm{T} 0} p_{T} \sqrt{p_{T}^{2}+m_{0}^{2}} \int_{y_{\min }}^{y_{\max }} \cosh y\left[1+\frac{q_{\mathrm{T}}-1}{T_{\mathrm{T}}} \sqrt{p_{T}^{2}+m_{0}^{2}} \cosh y\right]^{-\frac{q_{\mathrm{T}}}{q_{\mathrm{T}}-1}} d y
$$


where $C_{\mathrm{T} 0}$ is the normalization constant which gives $\int_{0}^{\infty} f_{\mathrm{T}}\left(p_{T}\right) d p_{T}=1, T_{\mathrm{T}}$ is the effective temperature, and $q_{\mathrm{T}}$ is the entropy index or nonequilibrium degree factor. Sometimes the upper index $q_{\mathrm{T}} /\left(q_{\mathrm{T}}-1\right)$ is replaced by $1 /\left(q_{\mathrm{T}}-1\right)$ due to $q_{\mathrm{T}}$ being very close to 1 and application of mean field approximation. The latter obtains a smaller $q_{\mathrm{T}}$.

Particularly, in the present work, we also use the two- or three-component standard distribution, which is different from the distributions introduced above. The standard Boltzmann, Fermi-Dirac, and Bose-Einstein distributions for the $i$ th component (group) can be uniformly shown as

$$
f_{i}\left(p_{T}\right)=\frac{1}{N} \frac{d N}{d p_{T}}=C_{i 0} p_{T} \sqrt{p_{T}^{2}+m_{0}^{2}} \int_{y_{\min }}^{y_{\max }} \cosh y\left[\exp \left(\frac{\sqrt{p_{T}^{2}+m_{0}^{2}} \cosh y}{T_{i}}\right)+S\right]^{-1} d y
$$

where $C_{i 0}$ is the normalization constant which gives $\int_{0}^{\infty} f_{i}\left(p_{T}\right) d p_{T}=1$ and $T_{i}$ is the effective temperature for the $i$ th component. In final state, the $p_{T}$ spectrum is contributed by the $l$ components of the distribution; that is

$$
f_{\mathrm{S}}\left(p_{T}\right)=\frac{1}{N} \frac{d N}{d p_{T}}=\sum_{i=1}^{l} k_{i} f_{i}\left(p_{T}\right),
$$

where $k_{i}$ is the relative weight contributed by the $i$ th component. This is the multi-component standard distribution. Considering the relative contribution of each component, we have the mean effective temperature to be

$$
T_{\mathrm{S}}=\sum_{i=1}^{l} k_{i} T_{i}
$$

where $T_{\mathrm{S}}$ reflects the mean excitation degree for different components and can be used to describe the effective temperature of emission source. We would like to point out that Eq. (6) is not a simple additive treatment for different temperatures, but an average weighted by different $k_{i}$, where $\sum_{i=1}^{l} k_{i}=1$.

It is expected that we can obtain the relation between the effective temperature $T\left(T_{\mathrm{T}-\mathrm{S}}, T_{\mathrm{T}}\right.$, or $\left.T_{\mathrm{S}}\right)$ and the particle's rest mass $m_{0}$. A linear fitting can obtain the intercept $T_{0}\left(T_{\mathrm{T}-\mathrm{S} 0}, T_{\mathrm{T} 0}\right.$, or $\left.T_{\mathrm{S} 0}\right)$ in linear relation $T-m_{0}$. As the temperature corresponds to massless $\left(m_{0}=0\right)$ particle, $T_{0}$ is regarded as the source real temperature, or the kinetic freeze-out temperature of interacting system. According to refs. [11, 27-30], we have the relation between $T$ and $T_{0}$,

$$
T=T_{0}+a m_{0} .
$$

According to refs. [27, 28], the slope $a$ can be given by $v_{0}^{2} / 2$ and $v_{0}$ is the (average and transverse) radial flow velocity which is valid for low $p_{T}$ only. Considering different distribution laws, $v_{0}$ can be $v_{\mathrm{T}-\mathrm{S} 0}$, $v_{\mathrm{T} 0}$, or $v_{\mathrm{S} 0}$, and $T_{0}$ can be $T_{\mathrm{T}-\mathrm{S} 0}, T_{\mathrm{T} 0}$, or $T_{\mathrm{S} 0}$, corresponding to Tsallis-standard, Tsallis, or standard distribution, respectively. In the above discussions, different distribution laws are in fact regarded as different types of "thermometers" or "thermometric scale" and "speedometers".

Since $a=v_{0}^{2} / 2$ in Eq. (7) is only valid for low $p_{T}$ region $[27,28]$, whereas the effective temperature is extracted in the present work for fits in $p_{T}$ range which goes beyond $2 \mathrm{GeV} / c$, we give up to extract radial flow $v_{0}$ from the slope $a$ in Eq. (7). Although other works $[11,29,30]$ also regard the intercept $T_{0}$ in Eq. (7) as the kinetic freeze-out temperature, different relations between radial flow velocity $v_{0}$ and slope $a$ are used, and in some cases the relation is undetermined. In view of uncertain relations between radial flow velocity and slope in wide $p_{T}$ range, we give up to extract radial flow velocity from the slope, and extract only the kinetic freeze-out temperature from $T_{0}$ in Eq. (7).

We need an alternative method to extract mean (transverse) flow velocity. In our very recent work [31], the linear relations between $T$ and $m_{0}$ [Eq. (7)], mean transverse momentum $\left\langle p_{T}\right\rangle$ and $m_{0}$, mean momentum $\langle p\rangle$ and $m_{0}, T$ and mean moving mass $\bar{m},\left\langle p_{T}\right\rangle$ and $\bar{m}$, as well as $\langle p\rangle$ and $\bar{m}$ are studied. From the analyses on dimension and quantity, we regard the intercept in $T-m_{0}$ relation [Eq. (7)] as the kinetic freeze-out temperature and the slope in $\left\langle p_{T}\right\rangle-\bar{m}$ (or $\langle p\rangle-\bar{m}$ ) relation as the mean transverse flow velocity $\left\langle u_{T}\right\rangle$ (or mean flow velocity $\langle u\rangle$ ). Particularly, for $\left\langle p_{T}\right\rangle-\bar{m}$ and $\langle p\rangle-\bar{m}$ relations, we have

$$
\left\langle p_{T}\right\rangle=\left\langle p_{T}\right\rangle_{0}+\left\langle u_{T}\right\rangle \bar{m}
$$

and

$$
\langle p\rangle=\langle p\rangle_{0}+\langle u\rangle \bar{m},
$$



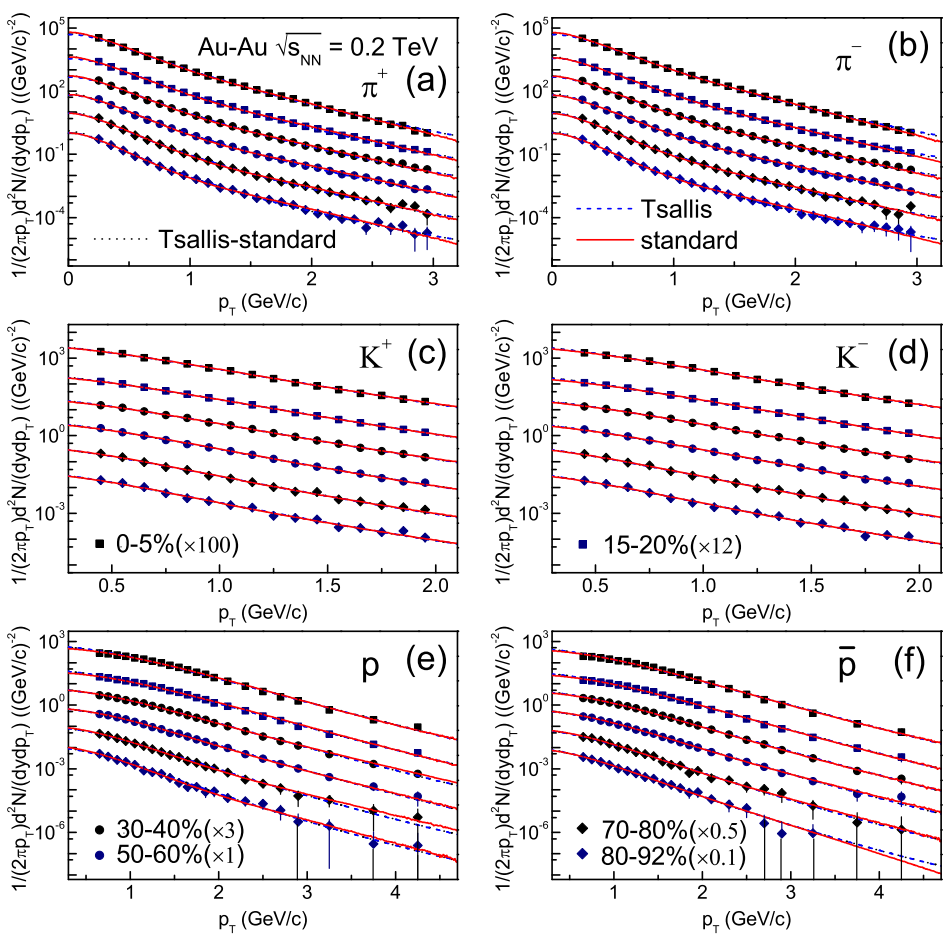

Figure 1. Transverse momentum spectra for (a) $\pi^{+}$, (b) $\pi^{-}$, (c) $K^{+}$, (d) $K^{-}$, (e) $p$, and (f) $\bar{p}$ produced in $\mathrm{Au}-\mathrm{Au}$ collisions with different centrality bins at $\sqrt{s_{N N}}=0.2 \mathrm{TeV}$. The data are measured by the PHENIX Collaboration at midrapidity [11] and are scaled vertically as quoted in the figure. The dotted, dashed, and solid curves are our results calculated by using the Tsallis-standard, Tsallis, and two-component standard distributions, respectively.

where $\left\langle p_{T}\right\rangle_{0}$ and $\langle p\rangle_{0}$ denote the mean transverse momentum and mean momentum of massless particle, respectively. We would like to point out that, in the case of describing meanwhile the same experimental data, the three distributions result in nearly the same $\left\langle p_{T}\right\rangle$ (or $\left.\langle p\rangle\right)$, the same $\left\langle p_{T}\right\rangle-\bar{m}$ (or $\left.\langle p\rangle-\bar{m}\right)$ relation, and the same $\left\langle u_{T}\right\rangle$ (or $\left.\langle u\rangle\right)$.

From the above analyses, we see that the linear relations between $T$ and $m_{0},\left\langle p_{T}\right\rangle$ and $\bar{m}$, as well as $\langle p\rangle$ and $\bar{m}$ [Eqs. (7)-(9)] can be respectively obtained from the same set of parameter values which are extracted from the same set of experimental data. This means that the extraction processes of $T_{0}$, $\left\langle u_{T}\right\rangle$, and $\langle u\rangle$ are independent, though their values are entangled due to the same set of parameters. In particular, $v_{0}$ and the slope in Eq. (7) are related to (transverse) flow velocity. However, there is no obvious and exact relation between them [11,27-30]. Anyhow, we think that the intercept in Eq. (7), the slope in Eq. (8), and the slope in Eq. (9) can provide a set of alternative methods to extract the kinetic freeze-out temperature, transverse flow velocity, and flow velocity, respectively [31]. Thus, we can use this set of alternative methods in the present work to extract separately the kinetic freeze-out temperature and transverse flow velocity according to transverse momentum spectra. This set of alternative methods is also used to judge different kinetic freeze-out scenarios in our another recent work [32] in which an evidence of mass-dependent differential kinetic freeze-out scenario is observed, while the single and double kinetic freeze-out scenarios are eliminated.

\section{Results and discussion}

Figure 1 presents the centrality dependence of $p_{T}$ spectra for (a) $\pi^{+}$, (b) $\pi^{-}$, (c) $K^{+}$, (d) $K^{-}$, 

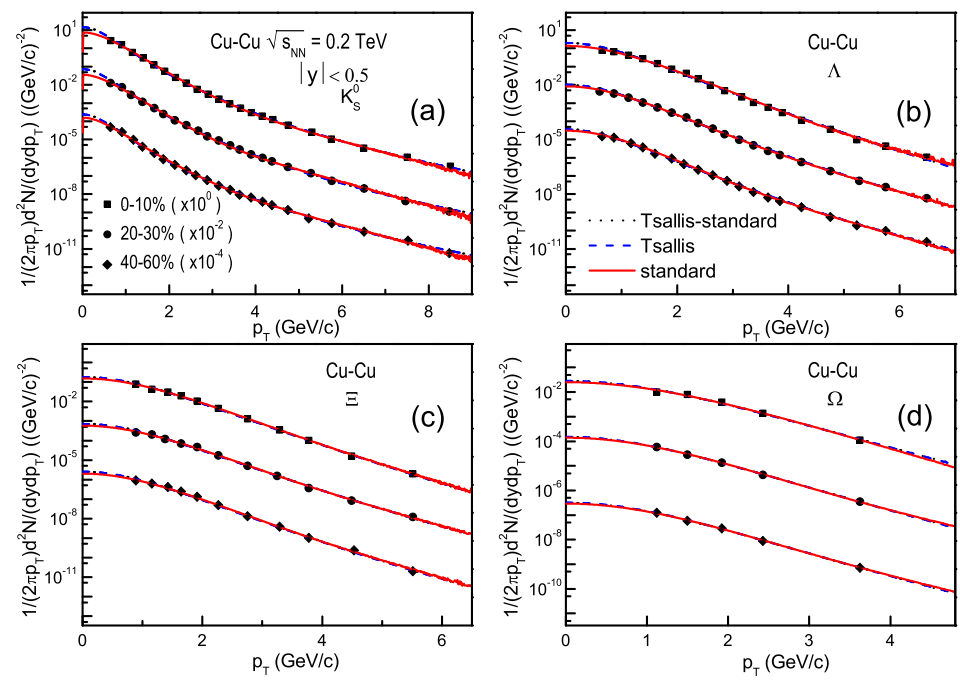

Figure 2. Transverse momentum spectra of (a) $K_{S}^{0}$, (b) $\Lambda$, (c) $\Xi$, and (d) $\Omega$ produced in $\mathrm{Cu}-\mathrm{Cu}$ collisions at $\sqrt{s_{N N}}=0.2 \mathrm{TeV}$ in three centrality intervals. The symbols represent the experimental data recorded by the STAR Collaboration in the rapidiy range $|y|<0.5$ [12]. The dotted, dashed, and solid curves are our results calculated by using the Tsallis-standard, Tsallis, and two- or three-component standard distributions, respectively.

(e) $p$, and (f) $\bar{p}$ produced in Au-Au collisions at center-of-mass energy $\sqrt{s_{N N}}=0.2 \mathrm{TeV}$. The data measured by the PHENIX Collaboration at midrapidity [11] are represented by different symbols which correspond to different centrality $(C)$ bins from $0-5 \%$ to $80-92 \%$ scaled by different amounts as shown in the panels for clarity. The error bars are statistical only. The dotted, dashed, and solid curves are our results calculated by using the Tsallis-standard, Tsallis, and two-component standard distributions, respectively. The values of free parameters $\left(T_{\mathrm{T}-\mathrm{S}}, q_{\mathrm{T}-\mathrm{S}}, T_{\mathrm{T}}\right.$, and $\left.q_{\mathrm{T}}\right)$, normalization constants $\left(N_{\mathrm{T}-\mathrm{S} 0}\right.$ and $\left.N_{\mathrm{T} 0}\right)$ for comparisons between curves and data, and $\chi^{2}$ per degree of freedom $\left(\chi^{2} /\right.$ dof $)$ for Tsallisstandard and Tsallis distributions are given in Table 1 . The values of free parameters $\left(T_{1}, k_{1}\right.$, and $\left.T_{2}\right)$, mean effective temperature $\left(T_{\mathrm{S}}\right)$, normalization constant $\left(N_{\mathrm{S} 0}\right)$ for comparisons between curves and data, and $\chi^{2} /$ dof for two-component standard distribution are listed in Table 2. One can see that all three types of distribution laws are consistent with the experimental data. The effective temperatures $T_{\mathrm{T}-\mathrm{S}}$, $T_{\mathrm{T}}$, and $T_{\mathrm{S}}$ increase with the increase of centrality or particle mass, and $T_{\mathrm{T}-\mathrm{S}} \leq T_{\mathrm{T}}<T_{\mathrm{S}}$ for a given set of data. We would like to point out that the increase of centrality and the decrease of centrality percentage have the same meaning.

Figure 2 shows $p_{T}$ spectra of (a) $K_{S}^{0}$, (b) $\Lambda$, (c) $\Xi$, and (d) $\Omega$ produced in $\mathrm{Cu}-\mathrm{Cu}$ collisions at $\sqrt{s_{N N}}=0.2 \mathrm{TeV}$ in different centrality intervals of $0-10 \%, 20-30 \%$, and $40-60 \%$. The symbols represent the experimental data recorded by the STAR Collaboration in the rapidiy range $|y|<0.5$ [12]. For clarity, the results for different $C$ intervals are scaled by different amounts shown in the panels. The uncertainties on the data points include both statistical and systematic errors. The dotted, dashed, and solid curves are our results based on the Tsallis-standard, Tsallis, and two- or three-component standard transverse momentum distributions, respectively. The values of free parameters $\left(T_{\mathrm{T}-\mathrm{S}}, q_{\mathrm{T}-\mathrm{S}}, T_{\mathrm{T}}, q_{\mathrm{T}}, T_{1}, k_{1}, T_{2}, k_{2}\right.$, and $\left.T_{3}\right), T_{\mathrm{S}}$, normalization constants $\left(N_{\mathrm{T}-\mathrm{S} 0}, N_{\mathrm{T} 0}\right.$, and $\left.N_{\mathrm{S} 0}\right)$, and $\chi^{2} /$ dof are displayed in Tables 1 and 2. Obviously, the experimental data can be described by the three types of fit functions for $p_{T}$ in all centrality bins. The effective temperatures $T_{\mathrm{T}-\mathrm{S}}, T_{\mathrm{T}}$, and $T_{\mathrm{S}}$ increase with the increase of centrality or particle mass, and $T_{\mathrm{T}-\mathrm{S}} \leq T_{\mathrm{T}}<T_{\mathrm{S}}$ for a given set of data.

The $p_{T}$ spectra of (a) $\pi^{+}$, (b) $K^{+}$, (c) $p$, and (d) $\phi$ produced in central (0-5\%), semi-central (50$60 \%$ ), and peripheral (80-90\%) Pb-Pb collisions at $\sqrt{s_{N N}}=2.76 \mathrm{TeV}$ are displayed in Fig. 3, where $N_{\mathrm{ev}}$ denotes the number of events. The data points are measured by the ALICE Collaboration at midrapidity $(|y|<0.5)[13,14]$. The error bars combined both statistical and systematic uncertainties. The fitting 

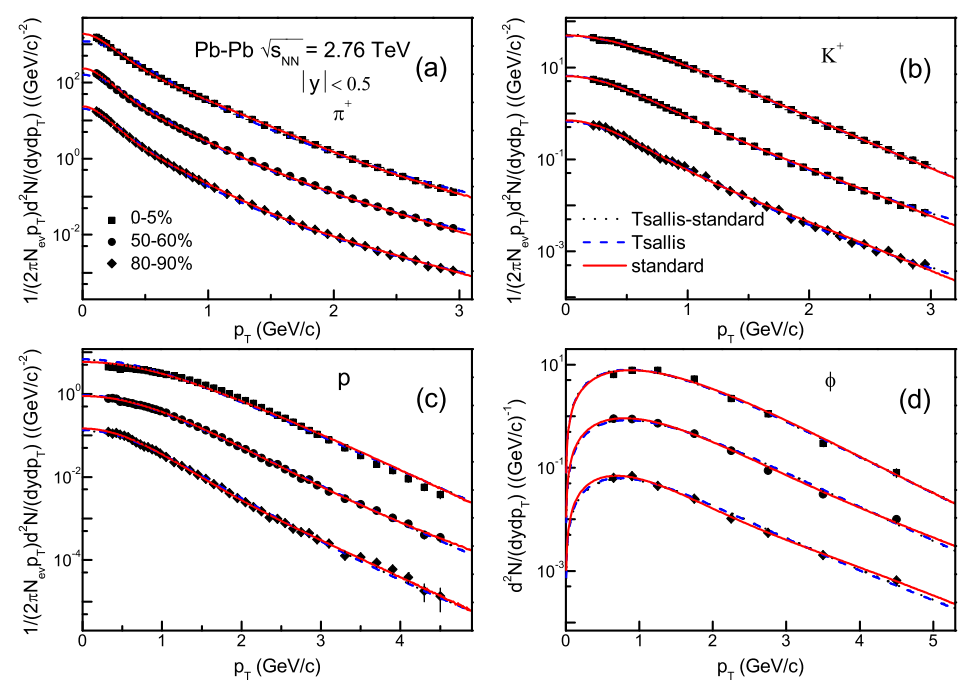

Figure 3. Transverse momentum spectra of (a) $\pi^{+}$, (b) $K^{+}$, (c) $p$, and (d) $\phi$ produced in $\mathrm{Pb}-\mathrm{Pb}$ collisions at $\sqrt{s_{N N}}=2.76 \mathrm{TeV}$ in three centrality intervals. The symbols represent the experimental data measured by the ALICE Collaboration at mid-rapidity $(|y|<0.5)[13,14]$. The fitting results with three types of distributions are plotted by the curves.
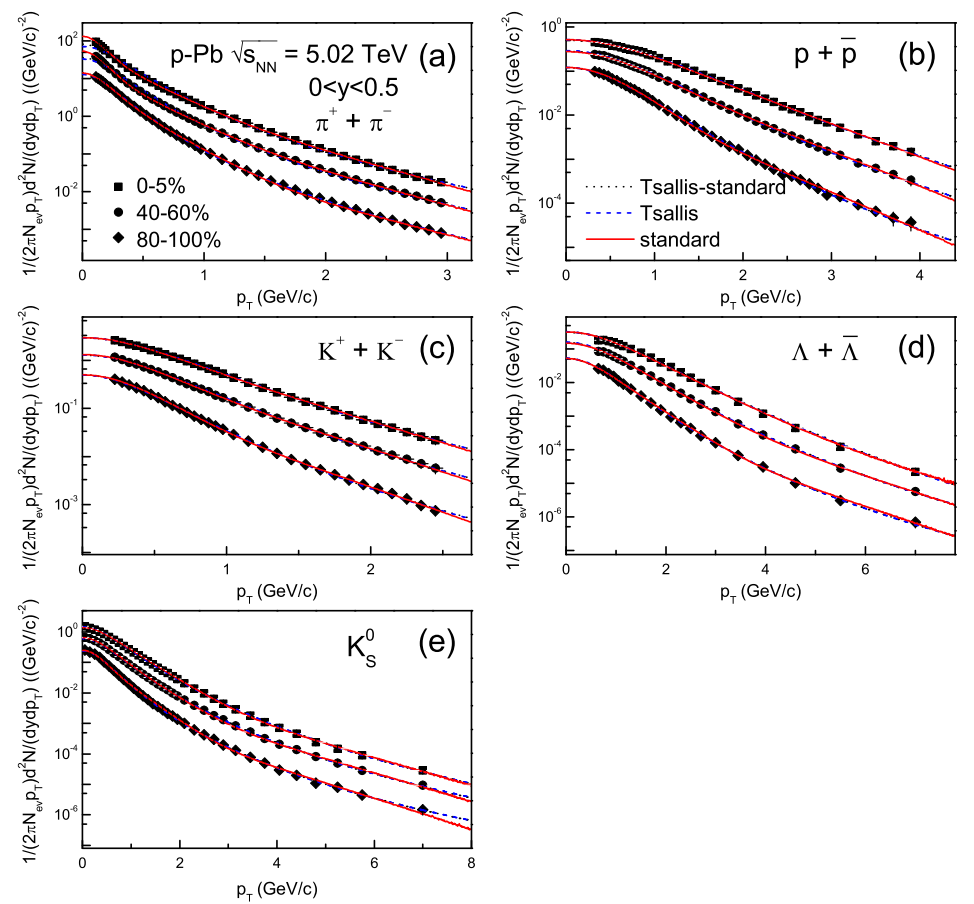

Figure 4. Transverse momentum spectra of (a) $\pi^{ \pm}$, (b) $p+\bar{p}$, (c) $K^{ \pm}$, (d) $\Lambda+\bar{\Lambda}$, and (e) $K_{S}^{0}$ in $p$ - $\mathrm{Pb}$ collisions in different centrality classes at $\sqrt{s_{N N}}=5.02 \mathrm{TeV}$. The experimental data represented by the symbols are performed by the ALICE Collaboration in $0<y<0.5$ [15]. Our fitting results are exhibited by the curves. 

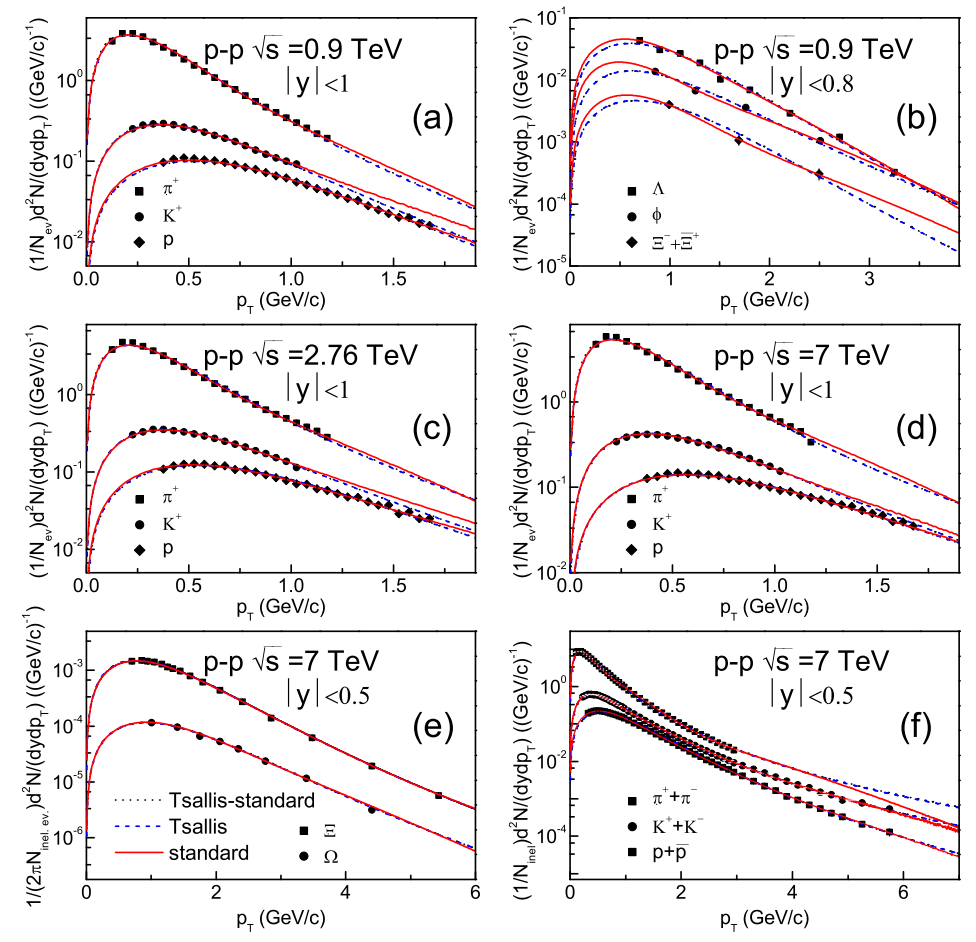

Figure 5. Transverse momentum spectra of various identified hadrons produced in $p-p$ collisions. The symbols in Figs. 5(a), 5(c), and 5(d) represent the experimental data for $\pi^{+}, K^{+}$, and $p$ measured by the CMS Collaboration [19] in the range $|y|<1$ at $\sqrt{s}=0.9,2.76,7 \mathrm{TeV}$, respectively. The data points of the ALICE Collaboration measured in $|y|<0.8$ at $\sqrt{s}=0.9 \mathrm{TeV}$ for $\Lambda, \phi$, and $\Xi^{-}+\bar{\Xi}^{+}[16]$, in $|y|<0.5$ at $\sqrt{s}=7 \mathrm{TeV}$ for $\Xi$ and $\Omega$ [17], and in $|y|<0.5$ at $\sqrt{s}=7 \mathrm{TeV}$ for $\pi^{ \pm}, K^{ \pm}$, and $p+\bar{p}[18]$ are shown in Figs. 5(b), 5(e), and 5(f), respectively. The fitting curves are our results.

results with Tsallis-standard, Tsallis, and two- or three-component standard distributions are plotted by the dotted, dashed, and solid curves, respectively. The values of free parameters, $T_{\mathrm{S}}$, normalization constants, and $\chi^{2} /$ dof are shown in Tables 1 and 2. These fitting functions describe the experimental data. Once again, the effective temperatures $T_{\mathrm{T}-\mathrm{S}}, T_{\mathrm{T}}$, and $T_{\mathrm{S}}$ increase with increase of centrality or particle mass, and $T_{\mathrm{T}-\mathrm{S}} \leq T_{\mathrm{T}}<T_{\mathrm{S}}$ for a given set of data.

Figure 4 exhibits $p_{T}$ spectra of (a) $\pi^{ \pm}$, (b) $p+\bar{p}$, (c) $K^{ \pm}$, (d) $\Lambda+\bar{\Lambda}$, and (e) $K_{S}^{0}$ produced in $p$ $\mathrm{Pb}$ collisions in different centrality classes $(0-5 \%, 40-60 \%$, and $80-100 \%)$ at $\sqrt{s_{N N}}=5.02 \mathrm{TeV}$. The experimental data represented by different symbols are performed by the ALICE Collaboration in the rapidity interval $0<y<0.5[15]$. The statistical and systematic uncertainties are combined in the error bars. Our results from the analyses of the Tsallis-standard, Tsallis, and two- or three-component standard distributions are exhibited by the dotted, dashed, and solid curves, respectively. The values of free parameters, $T_{S}$, normalization constants, and $\chi^{2} /$ dof are summarized in Tables 1 and 2 . As can be seen, the fitting results are in agreement with the experimental data. The effective temperatures $T_{\mathrm{T}-\mathrm{S}}$, $T_{\mathrm{T}}$, and $T_{\mathrm{S}}$ increase with the increase of centrality or particle mass, and $T_{\mathrm{T}-\mathrm{S}} \leq T_{\mathrm{T}}<T_{\mathrm{S}}$ for a given set of data.

Figure 5 gives $p_{T}$ spectra of various identified hadrons produced in $p$ - $p$ collisions at different energies, where $N_{\text {inel }}$ denotes the number of inelastic events. The symbols in Figs. 5(a), 5(c), and 5(d) represent the experimental data for $\pi^{+}, K^{+}$, and $p$ measured by the CMS Collaboration [19] in the range $|y|<1$ at $\sqrt{s}=0.9,2.76$, and $7 \mathrm{TeV}$, respectively. The data points of the ALICE Collaboration measured in $|y|<0.8$ at $\sqrt{s}=0.9 \mathrm{TeV}$ for $\Lambda, \phi$, and $\Xi^{-}+\bar{\Xi}^{+}[16]$, in $|y|<0.5$ at $\sqrt{s}=7 \mathrm{TeV}$ for $\Xi$ and $\Omega$ [17], and in $|y|<0.5$ at $\sqrt{s}=7 \mathrm{TeV}$ for $\pi^{ \pm}, K^{ \pm}$, and $p+\bar{p}$ [18] are shown in Figs. 5(b), 5(e), and 


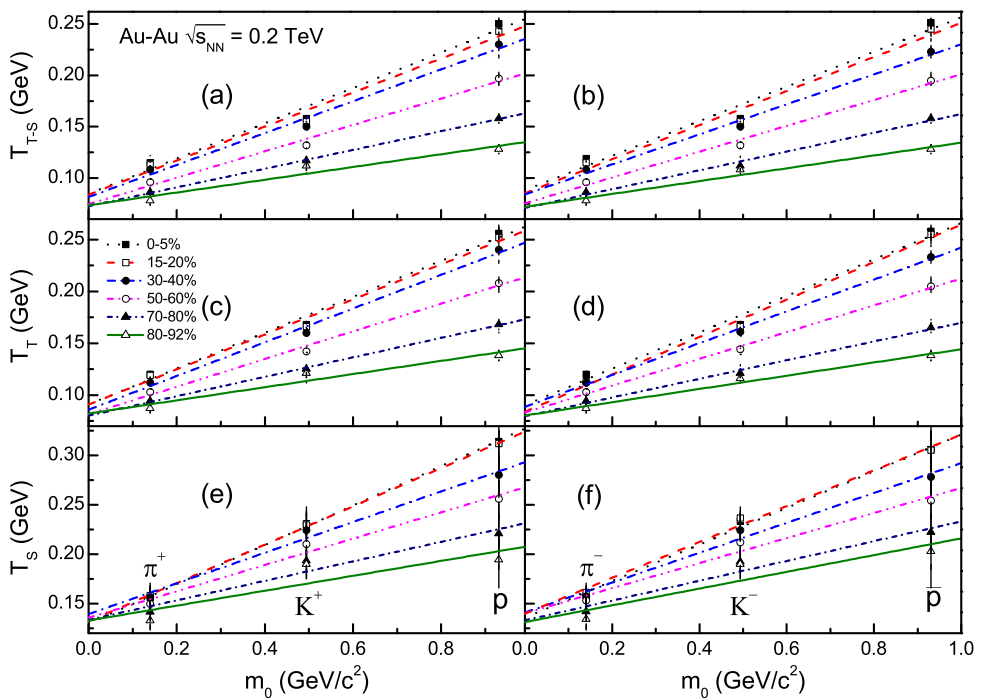

Figure 6. Particle mass and centrality dependences of $T_{\mathrm{T}-\mathrm{S}}, T_{\mathrm{T}}$, and $T_{\mathrm{S}}$ for positive [left: (a), (c), and (e)] and negative particles [right: (b), (d), and (f)] in Au-Au collisions at $\sqrt{s_{N N}}=0.2 \mathrm{TeV}$. The symbols represent the parameter values extracted from Fig. 1 and listed in Tables 1 and 2. The lines represent linear fits to the results from each centrality bin as a function of mass using Eq. (7).

$5(\mathrm{f})$, respectively. The uncertainties corresponding to combined statistics and systematics are shown as error bars. The fitting results (dotted, dashed, and solid curves) using mentioned functional forms (Tsallis-standard, Tsallis, and two- or three-component standard distributions) are superimposed. The values of free parameters, $T_{S}$, normalization constants, and $\chi^{2} /$ dof are exhibited in Tables 1 and 2 . Once more, the Tsallis-standard, Tsallis, and two- or three-component standard distributions can describe the experimental data of the considered particles. The effective temperatures $T_{\mathrm{T}-\mathrm{S}}, T_{\mathrm{T}}$, and $T_{\mathrm{S}}$ increase with the increase of particle mass, and $T_{\mathrm{T}-\mathrm{S}} \leq T_{\mathrm{T}}<T_{\mathrm{S}}$ for a given set of data.

To see clearly the dependences of effective temperatures $T_{\mathrm{T}-\mathrm{S}}, T_{\mathrm{T}}$, and $T_{\mathrm{S}}$ on centrality and particle mass, the related values listed in Tables 1 and 2, which are extracted from figures 1, 2, 3, and 4, are analyzed in Figs. 6, 7, 8 (left panel), and 8 (right panel), respectively. Different symbols correspond to different centrality intervals shown in the panels. The lines are our fitted results. At the same time, Fig. 9 shows the dependences of effective temperatures on particle mass for $p$ - $p$ collisions at the LHC energies. Different symbols correspond to different effective temperatures which are listed in Tables 1 and 2 and extracted from Fig. 5. The lines are our fitted results. Conclusions obtained from Figs. 1-5 and Tables 1 and 2 can be clearly seen from Figs. 6-9.

In Figs. 6-9, the lines are worthwhile for further investigation. In fact, according to Eq. (7), the intercepts at $m_{0}=0$ are real temperatures (kinetic freeze-out temperatures) $T_{\mathrm{T}-\mathrm{S} 0}, T_{\mathrm{T} 0}$, and $T_{\mathrm{S} 0}$, which are extracted from Tsallis-standard, Tsallis, and two- or three-component standard distributions, respectively. We show the dependences of kinetic freeze-out temperatures on centrality $C$ and energy $\sqrt{s_{N N}}$ in Fig. 10 for different sizes of collisions, where the related values for $p-p$ collisions are compared with central ( $0-5 \%)$ nucleus-nucleus collisions. In most cases, $T_{\mathrm{T}-\mathrm{S} 0} \leq T_{\mathrm{T} 0}<T_{\mathrm{S} 0}$. The kinetic freeze-out temperatures decrease slightly with the decrease of centrality (or with increase of centrality percentage), and do not show an obvious change with energy and size for the central, semi-central, and peripheral collisions. In the considered energy range from 0.2 to $7 \mathrm{TeV}$, the mean kinetic freeze-out temperatures $(\langle\ldots\rangle)$ extracted from different distribution laws for the mentioned three centralities are marked in the panels by the specific values and dashed lines. We see that $\left\langle T_{\mathrm{T}-\mathrm{S} 0}\right\rangle \leq\left\langle T_{\mathrm{T} 0}\right\rangle\left\langle\left\langle T_{\mathrm{S} 0}\right\rangle\right.$, and $\left.\left\langle T_{0}\right\rangle_{\text {central }}\right\rangle$ $\left\langle T_{0}\right\rangle_{\text {semi-central }} \geq\left\langle T_{0}\right\rangle_{\text {peripheral }}$. The independences of kinetic freeze-out temperatures on energy and size render that different interacting systems at the stage of kinetic freeze-out reached the same excitation degree of hadronic matter in the considered energy range.

We noticed that the value of kinetic freeze-out temperature $T_{\mathrm{S} 0}[(156 \pm 6) \mathrm{MeV}]$ in central collisions 


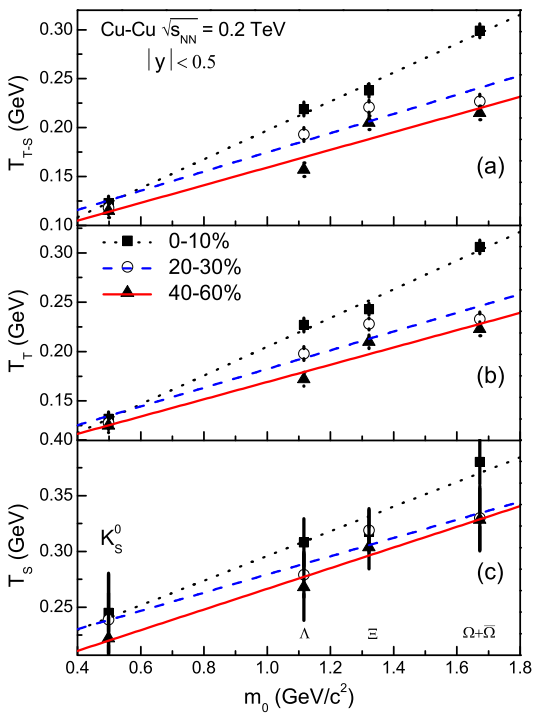

Figure 7. Same as Fig. 6, but for dependences of (a) $T_{\mathrm{T}-\mathrm{S}}$, (b) $T_{\mathrm{T}}$, and (c) $T_{\mathrm{S}}$ on particle mass and centrality in $\mathrm{Cu}-\mathrm{Cu}$ collisions at $\sqrt{s_{N N}}=0.2 \mathrm{TeV}$. The symbols represent the parameter values extracted from Fig. 2.
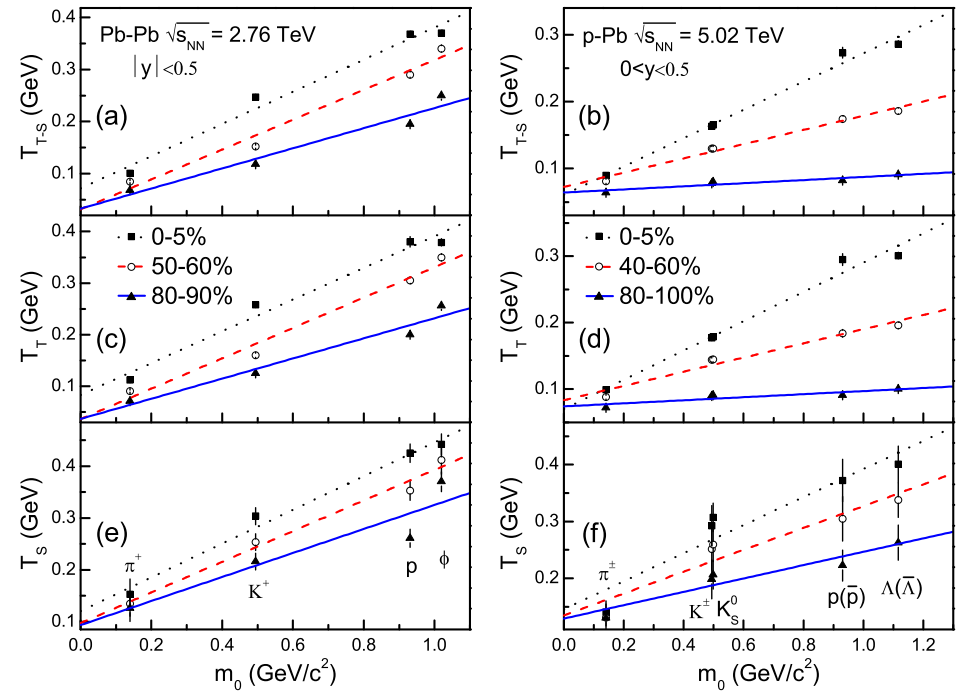

Figure 8. Same as Fig. 6, but for particle mass and centrality dependences of $T_{\mathrm{T}-\mathrm{S}}, T_{\mathrm{T}}$, and $T_{\mathrm{S}}$ for particles in $\mathrm{Pb}-\mathrm{Pb}$ collisions at $\sqrt{s_{N N}}=2.76 \mathrm{TeV}$ [left: (a), (c), and (e)], and in $p$-Pb collisions at $\sqrt{s_{N N}}=5.02 \mathrm{TeV}$ [right: (b), (d), and (f)]. The symbols represent the parameter values extracted from Figs. 3 and 4 . 

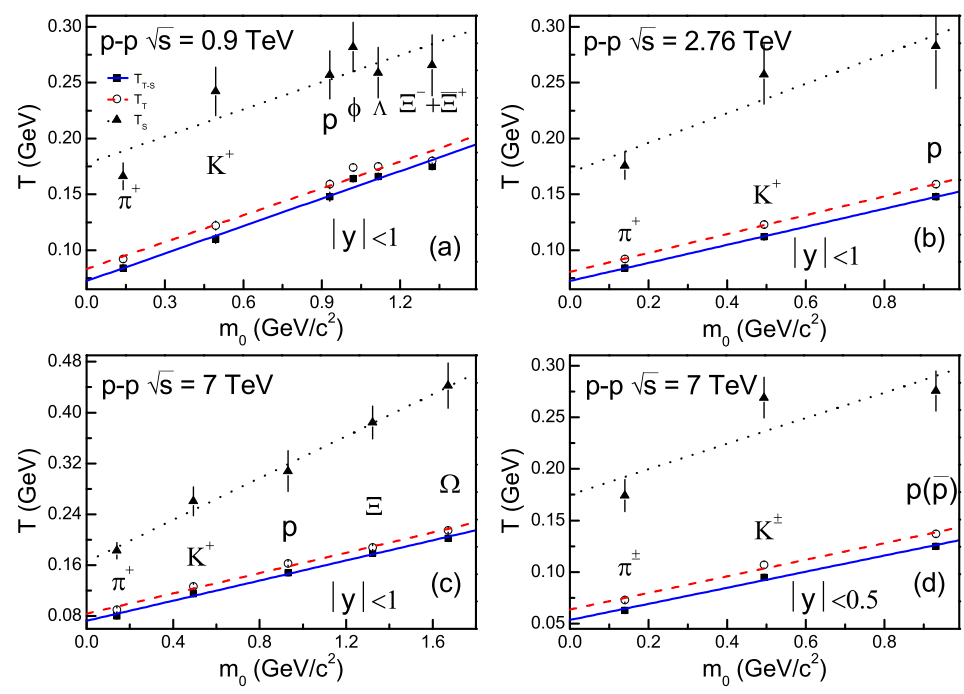

Figure 9. Same as Fig. 6, but for dependences of $T_{\mathrm{T}-\mathrm{S}}, T_{\mathrm{T}}$, and $T_{\mathrm{S}}$ on particle mass in $p-p$ collisions at (a) $\sqrt{s}=0.9 \mathrm{TeV}$, (b) $\sqrt{s}=2.76 \mathrm{TeV}$, (c) $\sqrt{s}=7 \mathrm{TeV}$ for positive particles, and (d) $\sqrt{s}=7 \mathrm{TeV}$ for charged particles. The symbols represent the parameter values extracted from Fig. 5.
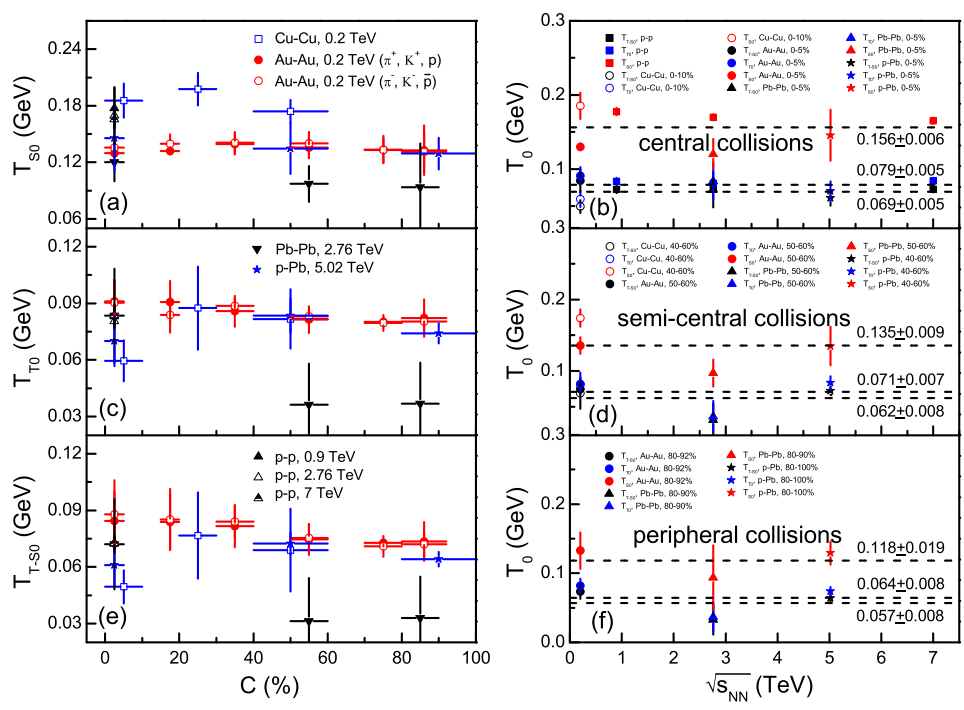

Figure 10. Centrality, energy, and size dependences of kinetic freeze-out temperatures $T_{\mathrm{T}-\mathrm{S} 0}, T_{\mathrm{T} 0}$, and $T_{\mathrm{S} 0}$. The left panel $[(\mathrm{a}),(\mathrm{c})$, and (e)] is mainly for different kinetic freeze-out temperatures and centralities, and the right panel [(b), (d), and (f)] is mainly for three centralities and different energies. The symbols represent our fitted results from Figs. 6-9. The results for $p-p$ collisions are plotted for comparisons with central (0-5\%) nucleus-nucleus collisions. The dashed lines marked with specific values in the right panel represent the three averages $\left\langle T_{\mathrm{T}-\mathrm{S} 0}\right\rangle,\left\langle T_{\mathrm{T} 0}\right\rangle$, and $\left\langle T_{\mathrm{S} 0}\right\rangle$ for three centralities, where $\left\langle T_{\mathrm{T}-\mathrm{S} 0}\right\rangle \leq\left\langle T_{\mathrm{T} 0}\right\rangle<$ $\left\langle T_{\mathrm{S} 0}\right\rangle$. 
[Fig. 10(b)] is less than that of chemical freeze-out temperature (170 MeV) used in some theoretical estimations for the critical point of QCD (quantum chromodynamics) [33-35]. Our result is also less than that of kinetic freeze-out temperature $(177 \mathrm{MeV})$ extracted from an exponential shape of transverse mass spectrum [11], and equal to that of chemical freeze-out temperature (156 MeV) extracted from particle ratios in a thermal and statistical model [36]. The latter item means that the kinetic freeze-out temperature obtained in the present work is greater than that obtained from the thermal and statistical model [36], or the kinetic and chemical freeze-outs had happened at almost the same time. We are inclined to the latter. These results are not exactly consistent with each other even if we consider the kinetic freeze-out temperature being less than the chemical freeze-out temperature. It is undoubted that more analyses are needed in the future.

To extract the transverse flow velocity, we analyze $\left\langle p_{T}\right\rangle-\bar{m}$ relations in Fig. 11, where the related parameters listed in Tables 1 and 2 are used to calculate $\left\langle p_{T}\right\rangle$ and $\bar{m}$, where the three distributions result in the same relations. Different symbols correspond to different centrality intervals shown in the panels. The lines are our fitted results. From the slopes, we can obtain the mean transverse flow velocities of particles produced in collisions with different centralities and at different energies. The related results are shown in Figs. 12(a) and 12(b) respectively, where the results for $p-p$ collisions are shown at $C=2.5 \%$ for comparisons. One can see that the mean transverse flow velocity in central collisions is greater than that in peripheral collisions. The relative difference is about $10 \%$.

The mean transverse flow velocity $\left\langle u_{T}\right\rangle[(0.443 \pm 0.071) c]$ for central collisions obtained in the present work is comparable with the radial flow velocity $[(0.426-0.472) c][37,38]$ of the blast-wave model $[7,37$, $38]$, while the value of $\left\langle u_{T}\right\rangle[(0.403 \pm 0.079) c]$ for peripheral collisions obtained in the present work is far from the radial flow velocity $(\approx 0)[37]$ of the blast-wave model $[7,37,38]$. In fact, $\left\langle u_{T}\right\rangle$ contains isotropic radial flow and anisotropic collective flow (directed flow and others). In central collisions, the effect of anisotropic collective flow is small [39], $\left\langle u_{T}\right\rangle$ contains mainly the isotropic radial flow. In peripheral collisions, the isotropic radial flow is nearly zero [37], $\left\langle u_{T}\right\rangle$ contains mainly the anisotropic collective flow [39]. According to Fig. 12(b), $\left\langle u_{T}\right\rangle$ in central collisions (which contains mainly the isotropic radial flow) is greater than that in peripheral collisions (which contains mainly the anisotropic collective flow).

Because of $\left\langle u_{T}\right\rangle$ containing the isotropic radial flow and anisotropic collective flow together, it is different from the original blast-wave model $[7,37,38]$ in which only the isotropic radial flow is contained. Our explanation on flow components is comparable with the improved blast-wave model $[40,41,42]$ in which the third parameter is introduced to describe the anisotropic transverse flow generated in noncentral collisions [40] and the fourth parameter is introduced to take into account the anisotropic shape of the source in coordinate space [41]. Although the dependence of elliptic flow on transverse momentum is described, the velocity of anisotropic transverse flow is not available in the improved blast-wave model $[40,41,42]$. As an indirect measurement of model independence, the present work is hoped to cause a further discussion on the flow velocity.

The kinetic freeze-out temperature and transverse flow velocity are extracted from the transverse momentum spectra, though the methodology used in the present work is different from that of the blastwave model with the Boltzmann-Gibbs distribution or the Tsallis statistics [7, 37, 38], or the quark recombination model with the Cooper-Frye formula and flow [43]. In the latter two models, the kinetic freeze-out temperature and radial flow velocity can be obtained from certain formula description. In the present work, we obtain the effective temperature from the formula of transverse momentum distribution, and kinetic freeze-out temperature from the intercept in linear $T-m_{0}$ relation [Eq. (7)] [11, 27, 29-32] and the transverse flow velocity from the slope in linear $\left\langle p_{T}\right\rangle-\bar{m}$ relation [Eq. (8)] [32]. The method used in the present work needs two steps to obtain the kinetic freeze-out temperature and transverse flow velocity, though the process of calculation is simple.

Although the topic discussed by us was intensively discussed in the literature and similar fitting and discussion were carried out in the literature many times, we have used an alternative methodology $[11,27$, 29-32] to extract the kinetic freeze-out temperature and transverse flow velocity, and the analysis is more extensive than anything done before. This methodology is different from that of the original blast-wave model $[7,37,38]$ or the quark recombination model [43]. In addition, differing from the original blastwave model which studies the kinetic freeze-out temperature and isotropic radial flow, the present work focuses on the kinetic freeze-out temperature and transverse flow which contains together the isotropic radial flow and anisotropic collective flow.

We would like to emphasize that our recent work [31] investigated particularly the methodology based on the analyses of transverse momentum spectra, in which the linear relations between $T$ and $m_{0},\left\langle p_{T}\right\rangle$ 

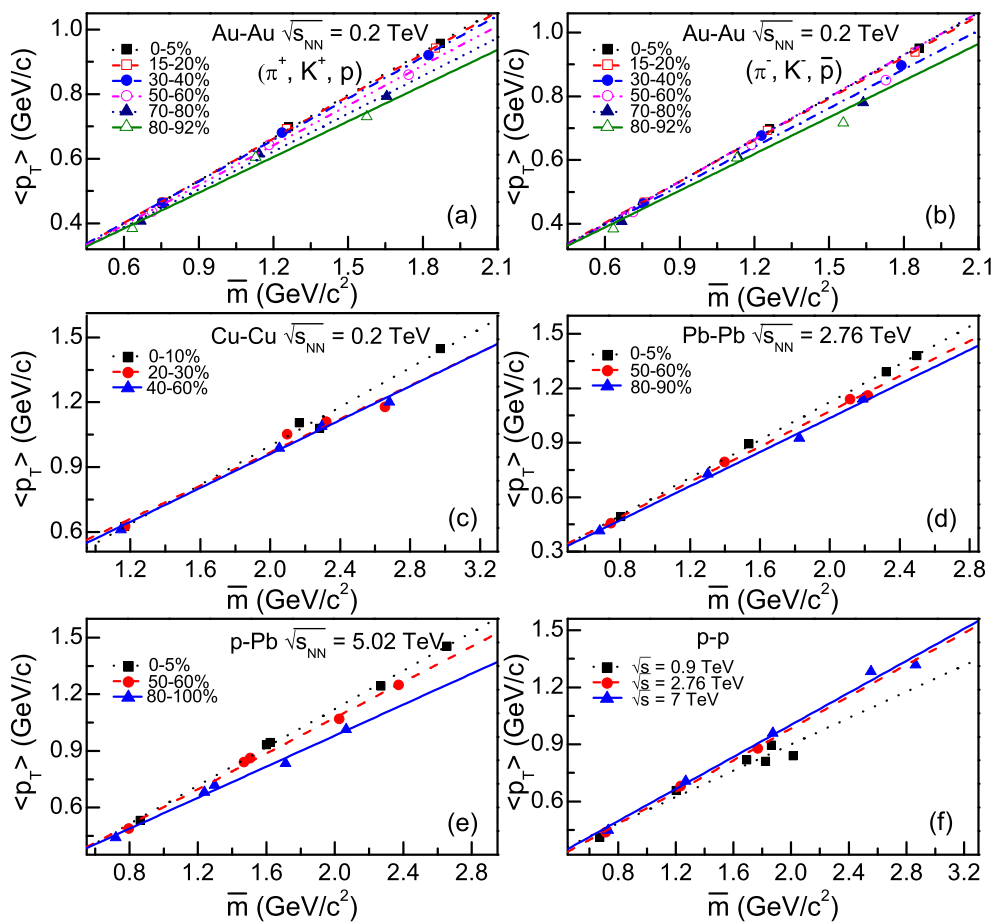

Figure 11. Dependences of $\left\langle p_{T}\right\rangle$ on $\bar{m}$ in (a) $0.2 \mathrm{TeV} \mathrm{Au}-\mathrm{Au}$ collisions for positive particles, (b) $0.2 \mathrm{TeV}$ $\mathrm{Au}-\mathrm{Au}$ collisions for negative particles, (c) $0.2 \mathrm{TeV} \mathrm{Cu}-\mathrm{Cu}$ collisions, (d) $2.76 \mathrm{TeV} \mathrm{Pb}-\mathrm{Pb}$ collisions, and (e) $5.02 \mathrm{TeV} p$-Pb collisions with different centralities, as well as (f) $p$ - $p$ collisions at different energies. The symbols are our calculated results due to the parameter values listed in Tables 1 and 2. The lines are our fitted results.

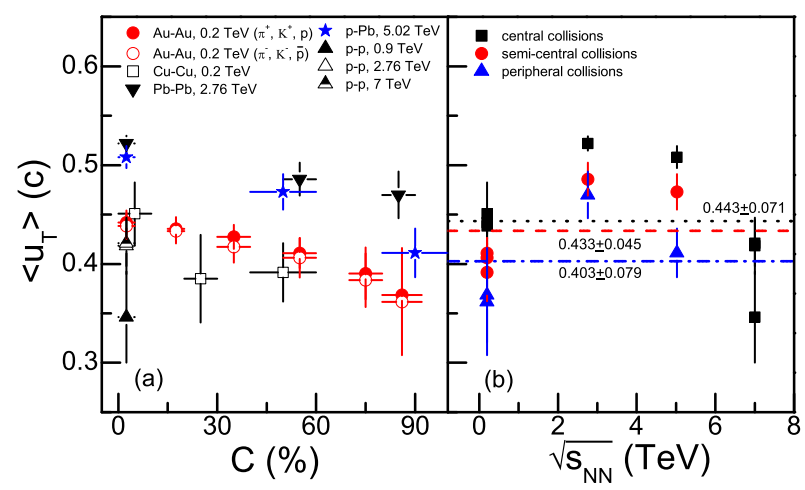

Figure 12. Dependences of $\left\langle u_{T}\right\rangle$ on (a) centrality and (b) energy for different collisions marked in the panel. The symbols are the slopes in Fig. 11. The dotted, dashed, and dotted-dashed lines in Fig. 12(b) are the mean $\left\langle u_{T}\right\rangle$ over energy for central, semi-central, and peripheral collisions, respectively. 
and $m_{0},\langle p\rangle$ and $m_{0}, T$ and $\bar{m},\left\langle p_{T}\right\rangle$ and $\bar{m}$, as well as $\langle p\rangle$ and $\bar{m}$ are studied. At the same time, the relations among some intercepts and slopes are studied. The present work focuses on an application of the above methodology. Different kinetic freeze-out temperatures are obtained due to different types of "thermometers" or "thermometric scales" and the same transverse flow velocity is obtained due to the same transverse momentum spectrum. Our descriptions on different "thermometers" or "thermometric scales" can be compared with those in thermodynamics.

In addition, the other difference is that in our recent work [31], the Tsallis distribution and a one- or two-component Erlang distribution is used, while in the present work, the Tsallis-standard, Tsallis, and two- or three-component standard distributions are used. The differences in the description of particle spectra with the three different distributions are marginal and only visible in a few cases at high momentum. This is also reflected in the similar values of the $\chi^{2} /$ dof as listed in Tables 1 and 2 of the present work. In our opinion, among different types of "thermometers" or "thermometric scales", the standard distribution and its multi-component distribution can be used to extract the standard temperature which is closest to the thermodynamic temperature in thermal physics. The other "thermometers" can be regarded as non-standard "thermometers" which can be corrected if necessary.

\section{Conclusions}

We summarize here our main observations and conclusions.

(a) The transverse momentum spectra of final-state light flavour particles $\left(\pi^{ \pm}, K^{ \pm}, K_{S}^{0}, p, \bar{p}, \phi, \Lambda\right.$, $\bar{\Lambda}, \Xi$, and $\Omega$, etc.), produced in $\mathrm{Au}-\mathrm{Au}, \mathrm{Cu}-\mathrm{Cu}, \mathrm{Pb}-\mathrm{Pb}, p-\mathrm{Pb}$, and $p-p$ collisions over an energy range from 0.2 to $7 \mathrm{TeV}$, are described by the Tsallis-standard, Tsallis, and two- or three-component standard distributions which are used in the multisource thermal model as different types of "thermometers" or "thermometric scales" and "speedometers". The characteristic of multiple temperatures in multicomponent standard distribution can be described by the Tsallis-standard and Tsallis distributions. The calculated results are in agreement with the experimental data recorded by the PHENIX, STAR, ALICE, and CMS Collaborations. Based on the three types of "thermometers" or "thermometric scales" and "speedometers", the effective temperatures $\left(T_{\mathrm{T}-\mathrm{S}}, T_{\mathrm{T}}\right.$, and $\left.T_{\mathrm{S}}\right)$ and kinetic freeze-out temperatures $\left(T_{\mathrm{T}-\mathrm{S} 0}, T_{\mathrm{T} 0}\right.$, and $\left.T_{\mathrm{S} 0}\right)$ of interacting system at the stage of kinetic freeze-out, and the mean transverse flow velocity of final-state particles are successively extracted from the transverse momentum spectra.

(b) In the extraction of the kinetic freeze-out temperature and transverse flow velocity from the transverse momentum spectra, the methodology used in the present work is different from that of the original blast-wave model with the Boltzmann-Gibbs distribution or the Tsallis statistics, or the quark recombination model with the Cooper-Frye formula and flow. The effect of transverse flow is not directly considered in formulas describing the transverse momentum spectra. Instead, we obtain the effective temperature from the formulas, the kinetic freeze-out temperature from the intercept by plotting the effective temperature versus the particle's rest mass, and the transverse flow velocity from the slope by plotting the mean transverse momentum versus the mean moving mass. Our treatment disentangles naturally the random thermal motion of particles and collective expansion of sources.

(c) The present work shows that the effective temperatures $T_{\mathrm{T}-\mathrm{S}}, T_{\mathrm{T}}$, and $T_{\mathrm{S}}$ increase with the increase of centrality or particle's rest mass, and satisfy the relationship $T_{\mathrm{T}-\mathrm{S}} \leq T_{\mathrm{T}}<T_{\mathrm{S}}$ for a given set of data. In most cases, we have $T_{\mathrm{T}-\mathrm{S} 0} \leq T_{\mathrm{T} 0}<T_{\mathrm{S} 0}$. The kinetic freeze-out temperatures decrease slightly with the decrease of centrality, and do not show an obvious change with energy and size for the central, semi-central, and peripheral collisions. The mean kinetic freeze-out temperatures derived from the three types of "thermometers" show $\left\langle T_{\mathrm{T}-\mathrm{S} 0}\right\rangle \leq\left\langle T_{\mathrm{T} 0}\right\rangle\left\langle\left\langle T_{\mathrm{S} 0}\right\rangle\right.$, and $\left\langle T_{0}\right\rangle_{\text {central }}>\left\langle T_{0}\right\rangle_{\text {semi-central }} \geq\left\langle T_{0}\right\rangle_{\text {peripheral }}$. The independences of kinetic freeze-out temperatures on energy and size reveal that different interacting systems at the stage of kinetic freeze-out reached the same excitation degree of hadronic matter in the considered energy range. Generally, the effective temperature is greater than kinetic freeze-out temperature, since the former containing both the thermal motion and flow effect while the latter containing only the thermal motion.

(d) The present work also shows that the central collisions have a higher kinetic freeze-out temperature than that of peripheral collisions. This is consistent with those of the chemical freeze-out temperature and effective temperature. Based on the three temperatures we conclude that the excitation degree of central collisions is higher than that of peripheral collisions at both the chemical and kinetic freeze-out stages. In addition, at the stage of kinetic freeze-out, the transverse flow velocity in central collisions is larger than that in peripheral collisions, indicating a larger squeeze and expansion in central collisions 
which also show a higher temperature and excitation degree. Thus, the kinetic freeze-out temperatures, which describe the random thermal motions of particles, and the transverse flow velocities, which describe the collective expansions of sources, paint a consistent picture.

(e) The present work is an application of our recent work [31] in which an alternative method is investigated to extract separately the kinetic freeze-out temperature and flow velocity. If we regard the standard distribution and its multi-component distribution as the most exact and standard "thermometer" due to they being closest to the thermodynamic temperature in thermal physics, we obtain a kinetic freeze-out temperature [(156 \pm 6$) \mathrm{MeV}$ in central collisions] which is equal to the chemical freeze-out temperature extracted from particle ratios [36]. This indicates that the two freeze-outs had happened at almost the same time even at the RHIC and LHC energies. We would like to treat other two "thermometers" used in the present work as non-standard "thermometers", which can be corrected if necessary. Another application [32] of our alternative method shows an evidence of mass-dependent differential kinetic freeze-out scenario, while the single and double kinetic freeze-out scenarios are eliminated.

(f) In central collisions, the transverse flow mainly contains the isotropic radial flow, since the effect of anisotropic collective flow is small and can be neglected in the extraction of transverse flow which contains mainly the isotropic radial flow. In contrast, the transverse flow of peripheral collisions includes mainly the anisotropic collective flow, since the radial flow is nearly zero. In this work, the transverse flow contains both the isotropic radial flow and anisotropic collective flow, which outperforms the original blast-wave model since it only includes the radial flow. We also found that the radial flow in central collisions is greater than anisotropic collective flow in peripheral collisions.

\section{Acknowledgments}

This work was supported by the National Natural Science Foundation of China under Grant No. 11575103 and the US DOE under contract DE-FG02-87ER40331.A008.

\section{References}

[1] Gupta N 2008 A Study of fluctuations in multiplicity distributions at ultrarelativistic heavy ion interactions, Ph.D. Thesis, University of Jammu, India, https://www.bnl.gov/userscenter/Thesis/2009/ngupta_thesis.pdf.

[2] Meng C-R 2009 Chin. Phys. Lett. 26102501.

[3] Liu F-H, Gao Y-Q and Wei H R 2014 Adv. High Energy Phys. 2014293873.

[4] Gao Y-Q, Tian C-X, Duan M-Y, Li B-C and Liu F-H 2012 Pramana - J. Phys. 791407.

[5] Wilk G and Wldarczyk Z 2000 Phys. Rev. Lett. 842770.

[6] Adams J et al. (STAR Collaboration) 2005 Phys. Rev. C 71064902.

[7] Schnedermann E, Sollfrank J and Heinz U 1993 Phys. Rev. C 482462.

[8] Liu F-H 2004 Phys. Lett. B 58368.

[9] Liu F-H 2008 Phys. Rev. C 78014902.

[10] Liu F-H 2008 Nucl. Phys. A 810159.

[11] Adler S S et al. (PHENIX Collaboration) 2004 Phys. Rev. C 69034909.

[12] Agakishiev G et al. (STAR Collaboration) 2012 Phys. Rev. Lett. 108072301.

[13] Abelev B et al. (ALICE Collaboration) 2013 Phys. Rev. C 88044910.

[14] Abelev B et al. (ALICE Collaboration) 2015 Phys. Rev. C 91024609.

[15] Abelev B et al. (ALICE Collaboration) 2014 Phys. Lett. B 72825. 
[16] Aamodt K et al. (ALICE Collaboration) 2011 Eur. Phys. J. C 711594.

[17] Floris M et al. (ALICE Collaboration) 2011 J. Phys. G 38124025.

[18] Adam J et al. (ALICE Collaboration) 2015 Eur. Phys. J. C 75226.

[19] Chatrchyan S et al. (CMS Collaboration) 2012 Eur. Phys. J. C 722164.

[20] Cleymans J and Worku D 2012 Eur. Phys. J. A 48160.

[21] Biró T S 2009 Eur. Phys. J. A 40255.

[22] Biró T S, Purcsel G and Ürmössy K 2009 Eur. Phys. J. A 40325.

[23] Tsallis C 1988 J. Stat. Phys. 52479.

[24] Tsallis C 2009 Braz. J. Phys. 39337.

[25] Biró T S 2001 Physica A 300424.

[26] Biró T S, Barnaföldi G G, Ván P and Ürmössy K 2014 arXiv:1404.1256 [hep-ph].

[27] Takeuchi S, Murase K, Hirano T, Huovinen P and Nara Y 2015 Phys. Rev. C 92044907.

[28] Heiselberg H and Levy A M 1999 Phys. Rev. C 592716.

[29] Heinz U W 2004 arXiv:hep-ph/0407360.

[30] Russo R 2015 Measurement of $D^{+}$meson production in $p$-Pb collisions with the ALICE detector, Ph.D. Thesis, Universitaâ degli Studi di Torino, Italy, arXiv:1511.04380 [nucl-ex].

[31] Wei H-R, Liu F-H and Lacey R A 2016 Eur. Phys. J. A 52102.

[32] Lao H-L, Wei H-R, Liu F-H and Lacey R A 2016 Eur. Phys. J. A 52203.

[33] Fodor Z and Katz S D 2004 JHEP 0404050.

[34] Datta S, Gavai R V and Gupta S 2013 Nucl. Phys. A 904-905 883c.

[35] Xu N (for the STAR Collaboration) 2014 Nucl. Phys. A 9311.

[36] Stachel J, Andronic A, Braun-Munzinger P and Redlich K 2014 J. Phys. Conf. Ser. 509012019.

[37] Tang Z B, Xu Y C, Ruan L J, van Buren G, Wang F Q and Xu Z B 2009 Phys. Rev. C 79, 051901(R).

[38] Shao M, Yi L, Tang Z B, Chen H F, Li C and Xu Z B 2010 J. Phys. G 37085104.

[39] Shi S S (for the STAR Collaboration) 2016 J. Phys. Conf. Ser. 668012079.

[40] Huovinen P, Kolb P F, Heinz U, Ruuskanen P V and Voloshin S 2001 Phys. Lett. B 50358.

[41] Adler C et al. (STAR Collaboration) 2001 Phys. Rev. Lett. 87182301.

[42] Sun X, Masui H, Poskanzer A M and Schmah A 2015 Phys. Rev. C 91024903.

[43] Ürmössy K and Biró T S 2010 Phys. Lett. B 68914. 
Table 1. Values of free parameters, normalization constants, and $\chi^{2} /$ dof corresponding to Tsallis-standard and Tsallis distributions in Figs. 1-5. The values of $\chi^{2} /$ dof for $\Xi^{-}+\bar{\Xi}^{+}$in Fig. 5(b) are in fact the values of $\chi^{2}$ values due to less data points. The temperatures are in the units of GeV.

\begin{tabular}{|c|c|c|c|c|c|c|c|c|c|}
\hline Figure & Type & $T_{\mathrm{T}-\mathrm{S}}$ & $q_{\mathrm{T}-\mathrm{S}}$ & $N_{\text {T-S0 }}$ & $\chi^{2} /$ dof & $T_{\mathrm{T}}$ & $q_{\mathrm{T}}$ & $N_{\text {T0 }}$ & $\overline{\chi^{2} / \text { dof }}$ \\
\hline \multirow[t]{6}{*}{$1(\mathrm{a})$} & $0-5 \%$ & $0.115 \pm 0.003$ & $1.076 \pm 0.004$ & $184.342 \pm 28.740$ & 0.243 & $0.120 \pm 0.003$ & $1.085 \pm 0.005$ & $169.870 \pm 25.534$ & 0.311 \\
\hline & $15-20 \%$ & $0.113 \pm 0.003$ & $1.080 \pm 0.004$ & $109.968 \pm 16.776$ & 0.193 & $0.119 \pm 0.003$ & $1.088 \pm 0.005$ & $102.208 \pm 17.748$ & 0.314 \\
\hline & $30-40 \%$ & $0.108 \pm 0.003$ & $1.086 \pm 0.004$ & $54.158 \pm 8.198$ & 0.347 & $0.112 \pm 0.003$ & $1.097 \pm 0.005$ & $52.515 \pm 7.948$ & 0.377 \\
\hline & $50-60 \%$ & $0.096 \pm 0.003$ & $1.093 \pm 0.004$ & $21.693 \pm 3.682$ & 0.273 & $0.103 \pm 0.003$ & $1.104 \pm 0.006$ & $20.256 \pm 3.357$ & 0.324 \\
\hline & $70-80 \%$ & $0.086 \pm 0.003$ & $1.097 \pm 0.004$ & $5.664 \pm 1.114$ & 0.234 & $0.094 \pm 0.003$ & $1.108 \pm 0.006$ & $5.234 \pm 1.008$ & 0.278 \\
\hline & $80-92 \%$ & $0.078 \pm 0.003$ & $1.101 \pm 0.005$ & $3.141 \pm 0.641$ & 0.149 & $0.087 \pm 0.003$ & $1.112 \pm 0.006$ & $2.870 \pm 0.584$ & 0.182 \\
\hline \multirow[t]{6}{*}{$1(\mathrm{~b})$} & $0-5 \%$ & $0.119 \pm 0.003$ & $1.072 \pm 0.004$ & $180.218 \pm 26.650$ & 0.190 & $0.120 \pm 0.003$ & $1.085 \pm 0.005$ & $169.269 \pm 24.783$ & 0.287 \\
\hline & $15-20 \%$ & $0.115 \pm 0.003$ & $1.078 \pm 0.004$ & $105.190 \pm 16.405$ & 0.181 & $0.113 \pm 0.003$ & $1.096 \pm 0.005$ & $102.669 \pm 15.936$ & 0.188 \\
\hline & $30-40 \%$ & $0.108 \pm 0.003$ & $1.086 \pm 0.004$ & $53.048 \pm 8.028$ & 0.271 & $0.112 \pm 0.003$ & $1.097 \pm 0.005$ & $51.420 \pm 7.818$ & 0.317 \\
\hline & $50-60 \%$ & $0.096 \pm 0.003$ & $1.093 \pm 0.004$ & $21.259 \pm 3.550$ & 0.220 & $0.103 \pm 0.003$ & $1.104 \pm 0.005$ & $19.834 \pm 3.249$ & 0.275 \\
\hline & $70-80 \%$ & $0.086 \pm 0.003$ & $1.097 \pm 0.004$ & $5.542 \pm 1.013$ & 0.223 & $0.094 \pm 0.003$ & $1.108 \pm 0.005$ & $5.120 \pm 0.965$ & 0.264 \\
\hline & $80-92 \%$ & $0.078 \pm 0.003$ & $1.101 \pm 0.005$ & $3.093 \pm 0.641$ & 0.099 & $0.087 \pm 0.003$ & $1.112 \pm 0.005$ & $2.826 \pm 0.584$ & 0.125 \\
\hline \multirow{6}{*}{$1(\mathrm{c})$} & $0-5 \%$ & $0.158 \pm 0.003$ & $1.066 \pm 0.005$ & $19.239 \pm 2.343$ & 0.066 & $0.168 \pm 0.003$ & $1.071 \pm 0.006$ & $18.916 \pm 2.664$ & 0.067 \\
\hline & $15-20 \%$ & $0.155 \pm 0.003$ & $1.067 \pm 0.004$ & $11.116 \pm 1.472$ & 0.023 & $0.166 \pm 0.003$ & $1.072 \pm 0.006$ & $10.851 \pm 1.426$ & 0.023 \\
\hline & $30-40 \%$ & $0.150 \pm 0.003$ & $1.068 \pm 0.004$ & $5.383 \pm 0.529$ & 0.031 & $0.160 \pm 0.003$ & $1.073 \pm 0.006$ & $5.308 \pm 0.757$ & 0.032 \\
\hline & $50-60 \%$ & $0.132 \pm 0.003$ & $1.074 \pm 0.005$ & $1.935 \pm 0.350$ & 0.050 & $0.142 \pm 0.003$ & $1.080 \pm 0.006$ & $1.914 \pm 0.339$ & 0.052 \\
\hline & $70-80 \%$ & $0.117 \pm 0.003$ & $1.080 \pm 0.006$ & $0.399 \pm 0.088$ & 0.132 & $0.125 \pm 0.003$ & $1.088 \pm 0.006$ & $0.397 \pm 0.082$ & 0.134 \\
\hline & $80-92 \%$ & $0.112 \pm 0.003$ & $1.082 \pm 0.006$ & $0.197 \pm 0.040$ & 0.203 & $0.121 \pm 0.003$ & $1.090 \pm 0.006$ & $0.198 \pm 0.048$ & 0.201 \\
\hline \multirow[t]{6}{*}{$1(\mathrm{~d})$} & $0-5 \%$ & $0.158 \pm 0.003$ & $1.066 \pm 0.005$ & $18.146 \pm 2.343$ & 0.040 & $0.168 \pm 0.003$ & $1.071 \pm 0.006$ & $17.850 \pm 2.664$ & 0.034 \\
\hline & $15-20 \%$ & $0.155 \pm 0.003$ & $1.067 \pm 0.004$ & $10.320 \pm 1.472$ & 0.041 & $0.166 \pm 0.003$ & $1.072 \pm 0.006$ & $10.067 \pm 1.426$ & 0.036 \\
\hline & $30-40 \%$ & $0.150 \pm 0.003$ & $1.067 \pm 0.004$ & $5.052 \pm 0.525$ & 0.030 & $0.161 \pm 0.003$ & $1.072 \pm 0.006$ & $4.956 \pm 0.514$ & 0.029 \\
\hline & $50-60 \%$ & $0.132 \pm 0.003$ & $1.075 \pm 0.005$ & $1.784 \pm 0.353$ & 0.059 & $0.144 \pm 0.003$ & $1.080 \pm 0.006$ & $1.746 \pm 0.312$ & 0.062 \\
\hline & $70-80 \%$ & $0.112 \pm 0.003$ & $1.083 \pm 0.006$ & $0.397 \pm 0.076$ & 0.085 & $0.121 \pm 0.003$ & $1.091 \pm 0.005$ & $0.379 \pm 0.068$ & 0.085 \\
\hline & $80-92 \%$ & $0.108 \pm 0.003$ & $1.086 \pm 0.006$ & $0.191 \pm 0.036$ & 0.178 & $0.116 \pm 0.003$ & $1.095 \pm 0.006$ & $0.193 \pm 0.042$ & 0.174 \\
\hline \multirow[t]{6}{*}{$1(\mathrm{e})$} & $0-5 \%$ & $0.250 \pm 0.005$ & $1.031 \pm 0.005$ & $4.940 \pm 0.820$ & 0.295 & $0.256 \pm 0.006$ & $1.032 \pm 0.005$ & $5.082 \pm 0.847$ & 0.304 \\
\hline & $15-20 \%$ & $0.243 \pm 0.005$ & $1.032 \pm 0.004$ & $2.929 \pm 0.460$ & 0.146 & $0.252 \pm 0.006$ & $1.033 \pm 0.004$ & $2.943 \pm 0.475$ & 0.150 \\
\hline & $30-40 \%$ & $0.230 \pm 0.006$ & $1.035 \pm 0.004$ & $1.493 \pm 0.302$ & 0.057 & $0.240 \pm 0.005$ & $1.036 \pm 0.005$ & $1.492 \pm 0.314$ & 0.059 \\
\hline & $50-60 \%$ & $0.197 \pm 0.006$ & $1.042 \pm 0.004$ & $0.559 \pm 0.107$ & 0.049 & $0.208 \pm 0.006$ & $1.043 \pm 0.006$ & $0.555 \pm 0.104$ & 0.052 \\
\hline & $70-80 \%$ & $0.158 \pm 0.004$ & $1.052 \pm 0.005$ & $0.126 \pm 0.032$ & 0.074 & $0.168 \pm 0.005$ & $1.054 \pm 0.005$ & $0.127 \pm 0.027$ & 0.076 \\
\hline & $80-92 \%$ & $0.128 \pm 0.004$ & $1.058 \pm 0.005$ & $0.070 \pm 0.023$ & 0.147 & $0.138 \pm 0.005$ & $1.061 \pm 0.005$ & $0.069 \pm 0.025$ & 0.150 \\
\hline \multirow[t]{6}{*}{$1(\mathrm{f})$} & $0-5 \%$ & $0.251 \pm 0.005$ & $1.029 \pm 0.004$ & $3.718 \pm 0.715$ & 0.413 & $0.258 \pm 0.006$ & $1.030 \pm 0.005$ & $3.771 \pm 0.742$ & 0.430 \\
\hline & $15-20 \%$ & $0.245 \pm 0.005$ & $1.030 \pm 0.004$ & $2.222 \pm 0.467$ & 0.231 & $0.255 \pm 0.006$ & $1.030 \pm 0.004$ & $2.218 \pm 0.490$ & 0.234 \\
\hline & $30-40 \%$ & $0.223 \pm 0.006$ & $1.034 \pm 0.004$ & $1.161 \pm 0.261$ & 0.085 & $0.233 \pm 0.006$ & $1.035 \pm 0.005$ & $1.153 \pm 0.274$ & 0.082 \\
\hline & $50-60 \%$ & $0.195 \pm 0.005$ & $1.041 \pm 0.004$ & $0.425 \pm 0.093$ & 0.079 & $0.205 \pm 0.006$ & $1.042 \pm 0.006$ & $0.426 \pm 0.080$ & 0.080 \\
\hline & $70-80 \%$ & $0.158 \pm 0.004$ & $1.049 \pm 0.005$ & $0.095 \pm 0.018$ & 0.119 & $0.165 \pm 0.005$ & $1.052 \pm 0.005$ & $0.096 \pm 0.024$ & 0.118 \\
\hline & $80-92 \%$ & $0.128 \pm 0.004$ & $1.055 \pm 0.005$ & $0.057 \pm 0.016$ & 0.099 & $0.138 \pm 0.005$ & $1.057 \pm 0.005$ & $0.057 \pm 0.018$ & 0.097 \\
\hline \multirow[t]{3}{*}{$2(\mathrm{a})$} & $0-10 \%$ & $0.123 \pm 0.007$ & $1.078 \pm 0.004$ & $7.085 \pm 2.401$ & 0.243 & $0.132 \pm 0.007$ & $1.085 \pm 0.004$ & $7.155 \pm 2.600$ & 0.242 \\
\hline & $20-30 \%$ & $0.118 \pm 0.007$ & $1.080 \pm 0.004$ & $3.580 \pm 1.194$ & 0.289 & $0.128 \pm 0.007$ & $1.087 \pm 0.004$ & $3.504 \pm 1.460$ & 0.290 \\
\hline & $40-60 \%$ & $0.115 \pm 0.007$ & $1.084 \pm 0.004$ & $1.181 \pm 0.450$ & 0.196 & $0.125 \pm 0.007$ & $1.092 \pm 0.004$ & $1.148 \pm 0.546$ & 0.195 \\
\hline \multirow[t]{3}{*}{$2(\mathrm{~b})$} & $0-10 \%$ & $0.219 \pm 0.007$ & $1.033 \pm 0.004$ & $1.624 \pm 0.722$ & 0.786 & $0.227 \pm 0.007$ & $1.034 \pm 0.004$ & $1.620 \pm 0.733$ & 0.789 \\
\hline & $20-30 \%$ & $0.193 \pm 0.007$ & $1.042 \pm 0.003$ & $0.778 \pm 0.303$ & 0.280 & $0.198 \pm 0.007$ & $1.045 \pm 0.004$ & $0.801 \pm 0.280$ & 0.275 \\
\hline & $40-60 \%$ & $0.157 \pm 0.006$ & $1.054 \pm 0.003$ & $0.278 \pm 0.104$ & 0.324 & $0.172 \pm 0.006$ & $1.055 \pm 0.004$ & $0.270 \pm 0.104$ & 0.331 \\
\hline \multirow[t]{3}{*}{$2(\mathrm{c})$} & $0-10 \%$ & $0.238 \pm 0.007$ & $1.031 \pm 0.003$ & $0.166 \pm 0.050$ & 0.165 & $0.243 \pm 0.006$ & $1.033 \pm 0.003$ & $0.167 \pm 0.048$ & 0.167 \\
\hline & $20-30 \%$ & $0.221 \pm 0.007$ & $1.039 \pm 0.003$ & $0.067 \pm 0.024$ & 0.382 & $0.228 \pm 0.006$ & $1.042 \pm 0.004$ & $0.065 \pm 0.023$ & 0.379 \\
\hline & $40-60 \%$ & $0.205 \pm 0.007$ & $0.040 \pm 0.003$ & $0.022 \pm 0.009$ & 0.295 & $0.210 \pm 0.006$ & $1.043 \pm 0.003$ & $0.022 \pm 0.008$ & 0.343 \\
\hline \multirow[t]{3}{*}{$2(d)$} & $0-10 \%$ & $0.299 \pm 0.007$ & $1.028 \pm 0.004$ & $0.032 \pm 0.009$ & 0.856 & $0.306 \pm 0.007$ & $1.029 \pm 0.005$ & $0.032 \pm 0.009$ & 0.858 \\
\hline & $20-30 \%$ & $0.227 \pm 0.007$ & $1.041 \pm 0.004$ & $0.015 \pm 0.003$ & 0.092 & $0.233 \pm 0.007$ & $1.044 \pm 0.004$ & $0.016 \pm 0.004$ & 0.089 \\
\hline & $40-60 \%$ & $0.215 \pm 0.007$ & $1.045 \pm 0.004$ & $0.003 \pm 0.001$ & 0.081 & $0.223 \pm 0.007$ & $1.048 \pm 0.004$ & $0.003 \pm 0.001$ & 0.080 \\
\hline
\end{tabular}


Table 1. Continued.

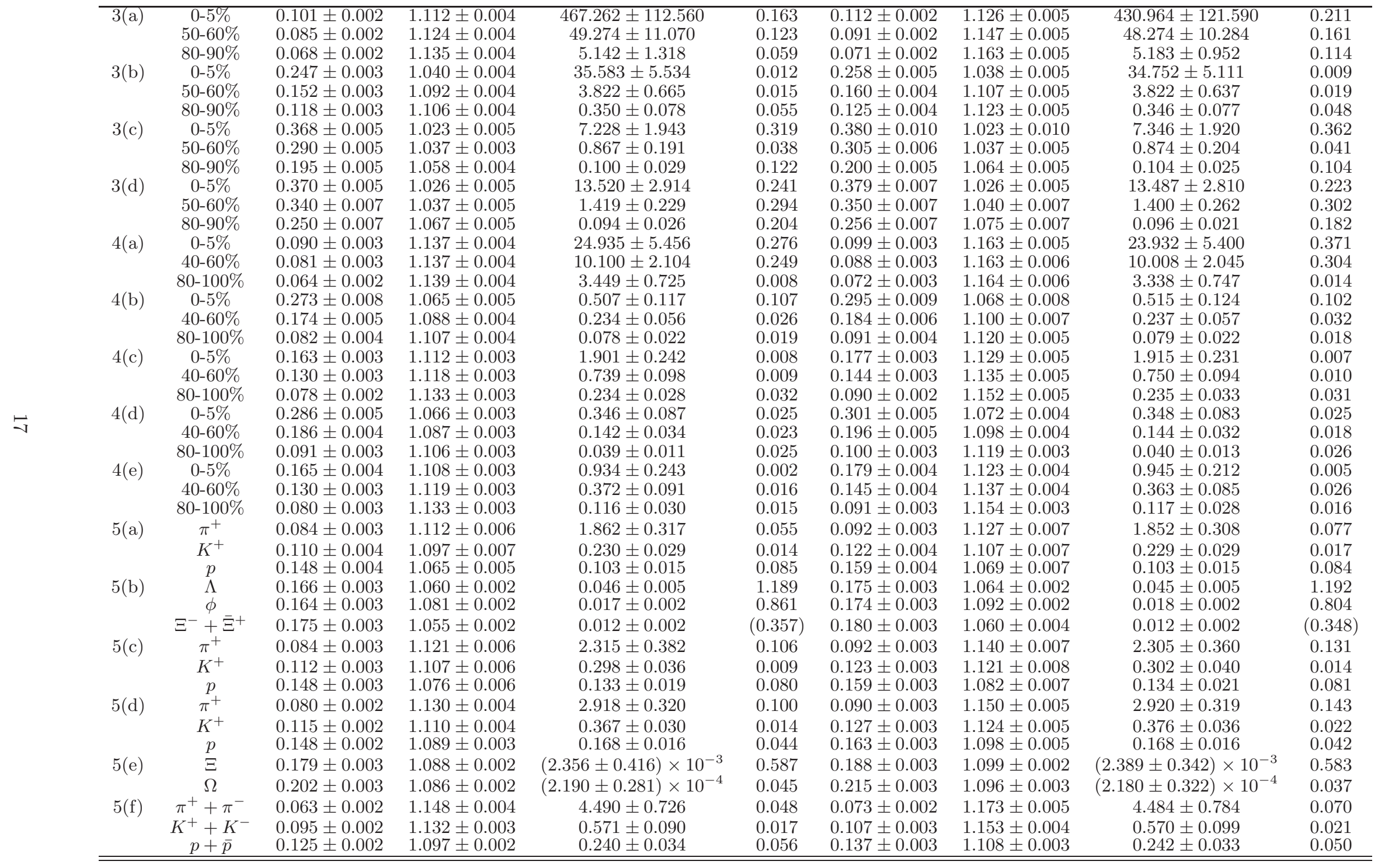


Table 2. Values of free parameters, $T_{\mathrm{S}}$, normalization constant, and $\chi^{2} /$ dof corresponding to two- or three-component standard distributions in Figs. $1-5$. The values of $\chi^{2} /$ dof for $\phi$ and $\Xi^{-}+\bar{\Xi}^{+}$in Fig. 5(b) are the values of $\chi^{2}$ values due to less data points. The temperatures are in the units of GeV.

\begin{tabular}{|c|c|c|c|c|c|c|c|c|c|}
\hline Figure & Type & $T_{1}$ & $k_{1}$ & $T_{2}$ & $k_{2}$ & $T_{3}$ & $T_{\mathrm{S}}$ & $N_{\mathrm{S} 0}$ & $\chi^{2} /$ dof \\
\hline \multirow[t]{6}{*}{$1(\mathrm{a})$} & $0-5 \%$ & $0.139 \pm 0.010$ & $0.874 \pm 0.020$ & $0.276 \pm 0.010$ & - & - & $0.156 \pm 0.010$ & $193.000 \pm 30.000$ & 0.306 \\
\hline & $15-20 \%$ & $0.142 \pm 0.010$ & $0.896 \pm 0.020$ & $0.298 \pm 0.010$ & - & - & $0.158 \pm 0.010$ & $109.000 \pm 15.000$ & 0.293 \\
\hline & $30-40 \%$ & $0.142 \pm 0.010$ & $0.913 \pm 0.010$ & $0.309 \pm 0.010$ & - & - & $0.156 \pm 0.010$ & $54.500 \pm 8.000$ & 0.457 \\
\hline & $50-60 \%$ & $0.137 \pm 0.008$ & $0.929 \pm 0.010$ & $0.315 \pm 0.010$ & - & - & $0.150 \pm 0.008$ & $20.500 \pm 3.000$ & 0.449 \\
\hline & $70-80 \%$ & $0.130 \pm 0.010$ & $0.935 \pm 0.010$ & $0.305 \pm 0.010$ & - & - & $0.141 \pm 0.010$ & $5.110 \pm 0.800$ & 0.344 \\
\hline & $80-92 \%$ & $0.121 \pm 0.010$ & $0.932 \pm 0.010$ & $0.291 \pm 0.010$ & - & - & $0.132 \pm 0.010$ & $2.800 \pm 0.400$ & 0.237 \\
\hline \multirow{6}{*}{ 1(b) } & $0-5 \%$ & $0.140 \pm 0.010$ & $0.860 \pm 0.020$ & $0.271 \pm 0.010$ & - & - & $0.158 \pm 0.010$ & $188.000 \pm 28.000$ & 0.269 \\
\hline & $15-20 \%$ & $0.145 \pm 0.010$ & $0.891 \pm 0.020$ & $0.293 \pm 0.010$ & - & - & $0.161 \pm 0.010$ & $102.000 \pm 13.000$ & 0.335 \\
\hline & $30-40 \%$ & $0.143 \pm 0.008$ & $0.913 \pm 0.010$ & $0.309 \pm 0.010$ & - & - & $0.157 \pm 0.008$ & $52.800 \pm 7.000$ & 0.483 \\
\hline & $50-60 \%$ & $0.140 \pm 0.010$ & $0.926 \pm 0.010$ & $0.315 \pm 0.010$ & - & - & $0.153 \pm 0.009$ & $19.320 \pm 2.000$ & 0.495 \\
\hline & $70-80 \%$ & $0.129 \pm 0.009$ & $0.926 \pm 0.010$ & $0.301 \pm 0.010$ & - & - & $0.142 \pm 0.008$ & $4.910 \pm 0.800$ & 0.354 \\
\hline & $80-92 \%$ & $0.122 \pm 0.010$ & $0.929 \pm 0.015$ & $0.291 \pm 0.020$ & - & - & $0.134 \pm 0.010$ & $2.700 \pm 0.500$ & 0.245 \\
\hline \multirow[t]{6}{*}{$1(\mathrm{c})$} & $0-5 \%$ & $0.207 \pm 0.010$ & $0.871 \pm 0.030$ & $0.390 \pm 0.030$ & - & - & $0.231 \pm 0.011$ & $18.400 \pm 2.000$ & 0.062 \\
\hline & $15-20 \%$ & $0.206 \pm 0.010$ & $0.866 \pm 0.030$ & $0.382 \pm 0.030$ & - & - & $0.230 \pm 0.011$ & $10.400 \pm 1.000$ & 0.026 \\
\hline & $30-40 \%$ & $0.202 \pm 0.010$ & $0.878 \pm 0.030$ & $0.385 \pm 0.030$ & - & - & $0.224 \pm 0.011$ & $5.060 \pm 0.500$ & 0.040 \\
\hline & $50-60 \%$ & $0.191 \pm 0.010$ & $0.887 \pm 0.030$ & $0.362 \pm 0.030$ & - & - & $0.210 \pm 0.011$ & $1.810 \pm 0.200$ & 0.093 \\
\hline & $70-80 \%$ & $0.169 \pm 0.010$ & $0.845 \pm 0.030$ & $0.325 \pm 0.025$ & - & - & $0.193 \pm 0.010$ & $0.389 \pm 0.080$ & 0.142 \\
\hline & $80-92 \%$ & $0.165 \pm 0.015$ & $0.840 \pm 0.030$ & $0.320 \pm 0.030$ & - & - & $0.190 \pm 0.014$ & $0.188 \pm 0.040$ & 0.200 \\
\hline \multirow[t]{6}{*}{$1(\mathrm{~d})$} & $0-5 \%$ & $0.210 \pm 0.010$ & $0.866 \pm 0.030$ & $0.384 \pm 0.030$ & - & - & $0.233 \pm 0.011$ & $16.800 \pm 2.000$ & 0.025 \\
\hline & $15-20 \%$ & $0.220 \pm 0.010$ & $0.912 \pm 0.030$ & $0.410 \pm 0.030$ & - & - & $0.237 \pm 0.011$ & $9.170 \pm 1.000$ & 0.055 \\
\hline & $30-40 \%$ & $0.202 \pm 0.010$ & $0.878 \pm 0.030$ & $0.385 \pm 0.030$ & - & - & $0.224 \pm 0.011$ & $4.710 \pm 0.500$ & 0.038 \\
\hline & $50-60 \%$ & $0.191 \pm 0.010$ & $0.877 \pm 0.030$ & $0.362 \pm 0.020$ & - & - & $0.212 \pm 0.010$ & $1.650 \pm 0.200$ & 0.074 \\
\hline & $70-80 \%$ & $0.169 \pm 0.010$ & $0.855 \pm 0.030$ & $0.330 \pm 0.025$ & - & - & $0.192 \pm 0.010$ & $0.378 \pm 0.080$ & 0.098 \\
\hline & $80-92 \%$ & $0.165 \pm 0.015$ & $0.840 \pm 0.040$ & $0.320 \pm 0.030$ & - & - & $0.190 \pm 0.018$ & $0.182 \pm 0.040$ & 0.184 \\
\hline \multirow[t]{6}{*}{$1(\mathrm{e})$} & $0-5 \%$ & $0.310 \pm 0.010$ & $0.980 \pm 0.020$ & $0.520 \pm 0.030$ & - & - & $0.314 \pm 0.011$ & $4.500 \pm 1.000$ & 0.216 \\
\hline & $15-20 \%$ & $0.300 \pm 0.020$ & $0.900 \pm 0.030$ & $0.420 \pm 0.030$ & - & - & $0.312 \pm 0.019$ & $2.600 \pm 0.600$ & 0.166 \\
\hline & $30-40 \%$ & $0.264 \pm 0.020$ & $0.900 \pm 0.030$ & $0.427 \pm 0.020$ & - & - & $0.280 \pm 0.018$ & $1.500 \pm 0.200$ & 0.061 \\
\hline & $50-60 \%$ & $0.240 \pm 0.020$ & $0.900 \pm 0.030$ & $0.400 \pm 0.030$ & - & - & $0.256 \pm 0.019$ & $0.560 \pm 0.100$ & 0.040 \\
\hline & $70-80 \%$ & $0.205 \pm 0.020$ & $0.920 \pm 0.030$ & $0.400 \pm 0.030$ & - & - & $0.221 \pm 0.019$ & $0.135 \pm 0.020$ & 0.032 \\
\hline & $80-92 \%$ & $0.180 \pm 0.030$ & $0.920 \pm 0.040$ & $0.360 \pm 0.030$ & - & - & $0.194 \pm 0.029$ & $0.076 \pm 0.015$ & 0.117 \\
\hline \multirow[t]{6}{*}{$1(\mathrm{f})$} & $0-5 \%$ & $0.300 \pm 0.020$ & $0.970 \pm 0.020$ & $0.490 \pm 0.030$ & - & - & $0.306 \pm 0.020$ & $3.500 \pm 0.800$ & 0.283 \\
\hline & $15-20 \%$ & $0.300 \pm 0.020$ & $0.970 \pm 0.020$ & $0.480 \pm 0.030$ & - & - & $0.305 \pm 0.020$ & $2.000 \pm 0.400$ & 0.155 \\
\hline & $30-40 \%$ & $0.265 \pm 0.020$ & $0.910 \pm 0.030$ & $0.410 \pm 0.030$ & - & - & $0.278 \pm 0.019$ & $1.100 \pm 0.200$ & 0.070 \\
\hline & $50-60 \%$ & $0.240 \pm 0.020$ & $0.910 \pm 0.030$ & $0.400 \pm 0.030$ & - & - & $0.254 \pm 0.019$ & $0.430 \pm 0.100$ & 0.059 \\
\hline & $70-80 \%$ & $0.205 \pm 0.020$ & $0.890 \pm 0.030$ & $0.360 \pm 0.020$ & - & - & $0.222 \pm 0.018$ & $0.100 \pm 0.030$ & 0.109 \\
\hline & $80-92 \%$ & $0.180 \pm 0.020$ & $0.810 \pm 0.050$ & $0.300 \pm 0.030$ & - & - & $0.203 \pm 0.018$ & $0.056 \pm 0.015$ & 0.104 \\
\hline \multirow[t]{3}{*}{$2(\mathrm{a})$} & $0-10 \%$ & $0.230 \pm 0.040$ & $0.881 \pm 0.001$ & $0.350 \pm 0.040$ & $0.117 \pm 0.001$ & $0.660 \pm 0.030$ & $0.245 \pm 0.036$ & $4.500 \pm 2.000$ & 0.490 \\
\hline & $20-30 \%$ & $0.217 \pm 0.050$ & $0.788 \pm 0.002$ & $0.315 \pm 0.040$ & $0.208 \pm 0.001$ & $0.624 \pm 0.030$ & $0.239 \pm 0.040$ & $2.100 \pm 0.900$ & 0.328 \\
\hline & $40-60 \%$ & $0.195 \pm 0.050$ & $0.792 \pm 0.001$ & $0.321 \pm 0.040$ & $0.205 \pm 0.001$ & $0.650 \pm 0.030$ & $0.222 \pm 0.040$ & $0.870 \pm 0.300$ & 0.206 \\
\hline \multirow[t]{3}{*}{$2(\mathrm{~b})$} & $0-10 \%$ & $0.305 \pm 0.020$ & $0.960 \pm 0.003$ & $0.360 \pm 0.030$ & $0.035 \pm 0.003$ & $0.550 \pm 0.030$ & $0.308 \pm 0.021$ & $1.250 \pm 0.300$ & 0.441 \\
\hline & $20-30 \%$ & $0.270 \pm 0.020$ & $0.932 \pm 0.003$ & $0.390 \pm 0.030$ & $0.060 \pm 0.003$ & $0.510 \pm 0.030$ & $0.279 \pm 0.020$ & $0.700 \pm 0.200$ & 0.208 \\
\hline & $40-60 \%$ & $0.260 \pm 0.030$ & $0.950 \pm 0.003$ & $0.400 \pm 0.030$ & $0.040 \pm 0.004$ & $0.500 \pm 0.030$ & $0.268 \pm 0.030$ & $0.250 \pm 0.100$ & 0.350 \\
\hline \multirow[t]{3}{*}{$2(\mathrm{c})$} & $0-10 \%$ & $0.310 \pm 0.020$ & $0.950 \pm 0.020$ & $0.465 \pm 0.020$ & - & - & $0.317 \pm 0.019$ & $0.150 \pm 0.050$ & 0.224 \\
\hline & $20-30 \%$ & $0.310 \pm 0.020$ & $0.950 \pm 0.020$ & $0.490 \pm 0.020$ & - & - & $0.319 \pm 0.019$ & $0.057 \pm 0.015$ & 0.336 \\
\hline & $40-60 \%$ & $0.295 \pm 0.020$ & $0.950 \pm 0.020$ & $0.470 \pm 0.020$ & - & - & $0.304 \pm 0.019$ & $0.019 \pm 0.005$ & 0.324 \\
\hline \multirow[t]{3}{*}{$2(\mathrm{~d})$} & $0-10 \%$ & $0.378 \pm 0.020$ & $0.990 \pm 0.010$ & $0.610 \pm 0.050$ & - & - & $0.380 \pm 0.019$ & $0.030 \pm 0.010$ & 1.170 \\
\hline & $20-30 \%$ & $0.316 \pm 0.030$ & $0.940 \pm 0.020$ & $0.550 \pm 0.050$ & - & - & $0.330 \pm 0.029$ & $0.015 \pm 0.005$ & 0.226 \\
\hline & $40-60 \%$ & $0.308 \pm 0.030$ & $0.900 \pm 0.030$ & $0.513 \pm 0.050$ & - & - & $0.328 \pm 0.028$ & $0.003 \pm 0.001$ & 0.203 \\
\hline
\end{tabular}


Table 2. Continued.

\begin{tabular}{|c|c|c|c|c|c|c|c|c|c|}
\hline \multirow[t]{3}{*}{$3(\mathrm{a})$} & $0-5 \%$ & $0.094 \pm 0.020$ & $0.650 \pm 0.005$ & $0.254 \pm 0.020$ & $0.341 \pm 0.005$ & $0.530 \pm 0.060$ & $0.152 \pm 0.030$ & $510.000 \pm 100.000$ & 0.025 \\
\hline & $50-60 \%$ & $0.083 \pm 0.020$ & $0.642 \pm 0.005$ & $0.208 \pm 0.020$ & $0.325 \pm 0.005$ & $0.413 \pm 0.020$ & $0.134 \pm 0.024$ & $57.000 \pm 10.000$ & 0.028 \\
\hline & $80-90 \%$ & $0.078 \pm 0.020$ & $0.635 \pm 0.005$ & $0.195 \pm 0.020$ & $0.342 \pm 0.005$ & $0.430 \pm 0.020$ & $0.126 \pm 0.026$ & $5.500 \pm 1.000$ & 0.021 \\
\hline \multirow[t]{3}{*}{$3(\mathrm{~b})$} & $0-5 \%$ & $0.275 \pm 0.020$ & $0.750 \pm 0.030$ & $0.389 \pm 0.020$ & - & - & $0.304 \pm 0.016$ & $35.000 \pm 5.000$ & 0.009 \\
\hline & $50-60 \%$ & $0.195 \pm 0.020$ & $0.680 \pm 0.040$ & $0.377 \pm 0.015$ & - & - & $0.253 \pm 0.016$ & $3.800 \pm 0.500$ & 0.019 \\
\hline & $80-90 \%$ & $0.160 \pm 0.020$ & $0.725 \pm 0.030$ & $0.363 \pm 0.015$ & - & - & $0.216 \pm 0.016$ & $0.360 \pm 0.060$ & 0.048 \\
\hline \multirow[t]{3}{*}{$3(\mathrm{c})$} & $0-5 \%$ & $0.447 \pm 0.020$ & $0.850 \pm 0.020$ & $0.300 \pm 0.020$ & - & - & $0.425 \pm 0.018$ & $6.800 \pm 2.000$ & 0.010 \\
\hline & $50-60 \%$ & $0.340 \pm 0.020$ & $0.950 \pm 0.010$ & $0.600 \pm 0.020$ & - & - & $0.353 \pm 0.019$ & $0.870 \pm 0.150$ & 0.019 \\
\hline & $80-90 \%$ & $0.228 \pm 0.020$ & $0.845 \pm 0.020$ & $0.440 \pm 0.020$ & - & - & $0.261 \pm 0.018$ & $0.110 \pm 0.020$ & 0.065 \\
\hline \multirow[t]{3}{*}{$3(d)$} & $0-5 \%$ & $0.430 \pm 0.020$ & $0.950 \pm 0.030$ & $0.670 \pm 0.050$ & - & - & $0.442 \pm 0.020$ & $13.500 \pm 3.000$ & 0.289 \\
\hline & $50-60 \%$ & $0.370 \pm 0.030$ & $0.850 \pm 0.030$ & $0.650 \pm 0.050$ & - & - & $0.412 \pm 0.028$ & $1.470 \pm 0.200$ & 0.157 \\
\hline & $80-90 \%$ & $0.290 \pm 0.020$ & $0.750 \pm 0.030$ & $0.610 \pm 0.030$ & - & - & $0.370 \pm 0.019$ & $0.100 \pm 0.020$ & 0.060 \\
\hline \multirow[t]{3}{*}{$4(\mathrm{a})$} & $0-5 \%$ & $0.068 \pm 0.020$ & $0.560 \pm 0.010$ & $0.200 \pm 0.020$ & $0.377 \pm 0.010$ & $0.430 \pm 0.020$ & $0.140 \pm 0.014$ & $30.560 \pm 7.000$ & 0.024 \\
\hline & $40-60 \%$ & $0.071 \pm 0.020$ & $0.625 \pm 0.010$ & $0.205 \pm 0.020$ & $0.335 \pm 0.010$ & $0.443 \pm 0.020$ & $0.131 \pm 0.015$ & $12.000 \pm 3.000$ & 0.037 \\
\hline & $80-100 \%$ & $0.085 \pm 0.020$ & $0.632 \pm 0.005$ & $0.195 \pm 0.015$ & $0.352 \pm 0.004$ & $0.478 \pm 0.020$ & $0.130 \pm 0.030$ & $3.450 \pm 0.800$ & 0.049 \\
\hline \multirow[t]{3}{*}{$4(\mathrm{~b})$} & $0-5 \%$ & $0.283 \pm 0.050$ & $0.608 \pm 0.080$ & $0.510 \pm 0.030$ & - & - & $0.372 \pm 0.037$ & $0.510 \pm 0.100$ & 0.117 \\
\hline & $40-60 \%$ & $0.240 \pm 0.050$ & $0.730 \pm 0.050$ & $0.480 \pm 0.030$ & - & - & $0.305 \pm 0.039$ & $0.230 \pm 0.050$ & 0.036 \\
\hline & $80-100 \%$ & $0.190 \pm 0.030$ & $0.873 \pm 0.030$ & $0.445 \pm 0.030$ & - & - & $0.222 \pm 0.027$ & $0.080 \pm 0.020$ & 0.060 \\
\hline \multirow[t]{3}{*}{$4(\mathrm{c})$} & $0-5 \%$ & $0.196 \pm 0.050$ & $0.556 \pm 0.080$ & $0.413 \pm 0.030$ & - & - & $0.292 \pm 0.035$ & $1.900 \pm 0.400$ & 0.019 \\
\hline & $40-60 \%$ & $0.175 \pm 0.050$ & $0.640 \pm 0.080$ & $0.388 \pm 0.030$ & - & - & $0.252 \pm 0.038$ & $0.750 \pm 0.200$ & 0.018 \\
\hline & $80-100 \%$ & $0.150 \pm 0.040$ & $0.770 \pm 0.070$ & $0.360 \pm 0.020$ & - & - & $0.198 \pm 0.034$ & $0.240 \pm 0.060$ & 0.042 \\
\hline \multirow[t]{3}{*}{$4(d)$} & $0-5 \%$ & $0.310 \pm 0.050$ & $0.590 \pm 0.004$ & $0.520 \pm 0.030$ & $0.400 \pm 0.004$ & $0.982 \pm 0.050$ & $0.401 \pm 0.032$ & $0.350 \pm 0.070$ & 0.085 \\
\hline & $40-60 \%$ & $0.250 \pm 0.050$ & $0.540 \pm 0.005$ & $0.424 \pm 0.030$ & $0.442 \pm 0.005$ & $0.842 \pm 0.040$ & $0.338 \pm 0.030$ & $0.140 \pm 0.030$ & 0.094 \\
\hline & $80-100 \%$ & $0.180 \pm 0.050$ & $0.563 \pm 0.003$ & $0.358 \pm 0.030$ & $0.426 \pm 0.003$ & $0.800 \pm 0.040$ & $0.263 \pm 0.031$ & $0.040 \pm 0.010$ & 0.147 \\
\hline \multirow[t]{3}{*}{$4(\mathrm{e})$} & $0-5 \%$ & $0.210 \pm 0.050$ & $0.451 \pm 0.002$ & $0.370 \pm 0.020$ & $0.530 \pm 0.002$ & $0.862 \pm 0.030$ & $0.307 \pm 0.025$ & $0.940 \pm 0.200$ & 0.006 \\
\hline & $40-60 \%$ & $0.180 \pm 0.050$ & $0.576 \pm 0.002$ & $0.348 \pm 0.020$ & $0.407 \pm 0.002$ & $0.840 \pm 0.030$ & $0.260 \pm 0.030$ & $0.380 \pm 0.070$ & 0.006 \\
\hline & $80-100 \%$ & $0.130 \pm 0.040$ & $0.598 \pm 0.002$ & $0.305 \pm 0.020$ & $0.390 \pm 0.002$ & $0.780 \pm 0.030$ & $0.206 \pm 0.025$ & $0.120 \pm 0.020$ & 0.012 \\
\hline \multirow[t]{3}{*}{$5(\mathrm{a})$} & $\pi^{+}$ & $0.124 \pm 0.010$ & $0.738 \pm 0.050$ & $0.285 \pm 0.020$ & - & - & $0.166 \pm 0.012$ & $1.870 \pm 0.200$ & 0.069 \\
\hline & $K^{+}$ & $0.165 \pm 0.010$ & $0.610 \pm 0.070$ & $0.363 \pm 0.040$ & - & - & $0.242 \pm 0.022$ & $0.235 \pm 0.030$ & 0.030 \\
\hline & $p$ & $0.187 \pm 0.020$ & $0.637 \pm 0.050$ & $0.380 \pm 0.040$ & - & - & $0.257 \pm 0.022$ & $0.106 \pm 0.010$ & 0.027 \\
\hline \multirow[t]{3}{*}{$5(\mathrm{~b})$} & $\Lambda$ & $0.188 \pm 0.030$ & $0.610 \pm 0.060$ & $0.370 \pm 0.020$ & - & - & $0.260 \pm 0.023$ & $0.050 \pm 0.005$ & 1.431 \\
\hline & $\phi$ & $0.170 \pm 0.020$ & $0.600 \pm 0.060$ & $0.450 \pm 0.020$ & - & - & $0.282 \pm 0.022$ & $0.021 \pm 0.002$ & $(0.371)$ \\
\hline & $\Xi^{-}+\bar{\Xi}^{+}$ & $0.190 \pm 0.030$ & $0.730 \pm 0.050$ & $0.470 \pm 0.030$ & - & - & $0.266 \pm 0.030$ & $0.013 \pm 0.001$ & $(0.014)$ \\
\hline \multirow[t]{3}{*}{$5(\mathrm{c})$} & $\pi^{+}$ & $0.122 \pm 0.010$ & $0.670 \pm 0.050$ & $0.285 \pm 0.020$ & - & - & $0.176 \pm 0.012$ & $2.300 \pm 0.300$ & 0.068 \\
\hline & $K^{+}$ & $0.167 \pm 0.020$ & $0.550 \pm 0.080$ & $0.368 \pm 0.040$ & - & - & $0.257 \pm 0.026$ & $0.305 \pm 0.040$ & 0.014 \\
\hline & $p$ & $0.189 \pm 0.030$ & $0.555 \pm 0.100$ & $0.400 \pm 0.060$ & - & - & $0.283 \pm 0.038$ & $0.137 \pm 0.020$ & 0.043 \\
\hline \multirow[t]{3}{*}{$5(\mathrm{~d})$} & $\pi^{+}$ & $0.122 \pm 0.010$ & $0.650 \pm 0.050$ & $0.296 \pm 0.020$ & - & - & $0.183 \pm 0.013$ & $2.900 \pm 0.300$ & 0.094 \\
\hline & $K^{+}$ & $0.169 \pm 0.010$ & $0.540 \pm 0.060$ & $0.368 \pm 0.040$ & - & - & $0.260 \pm 0.022$ & $0.375 \pm 0.030$ & 0.019 \\
\hline & $p$ & $0.210 \pm 0.020$ & $0.510 \pm 0.090$ & $0.410 \pm 0.050$ & - & - & $0.308 \pm 0.032$ & $0.167 \pm 0.015$ & 0.043 \\
\hline \multirow[t]{2}{*}{$5(\mathrm{e})$} & $\Xi$ & $0.270 \pm 0.040$ & $0.510 \pm 0.010$ & $0.470 \pm 0.030$ & $0.450 \pm 0.010$ & $0.880 \pm 0.030$ & $0.384 \pm 0.026$ & $(2.350 \pm 0.300) \times 10^{-3}$ & 0.733 \\
\hline & $\Omega$ & $0.340 \pm 0.040$ & $0.680 \pm 0.050$ & $0.660 \pm 0.050$ & - & - & $0.442 \pm 0.035$ & $(2.200 \pm 0.300) \times 10^{-4}$ & 0.084 \\
\hline \multirow[t]{3}{*}{$5(\mathrm{f})$} & $\pi^{+}+\pi^{-}$ & $0.096 \pm 0.020$ & $0.584 \pm 0.010$ & $0.254 \pm 0.020$ & $0.384 \pm 0.010$ & $0.650 \pm 0.040$ & $0.174 \pm 0.016$ & $4.480 \pm 0.800$ & 0.061 \\
\hline & $K^{+}+K^{-}$ & $0.160 \pm 0.030$ & $0.550 \pm 0.009$ & $0.358 \pm 0.020$ & $0.405 \pm 0.009$ & $0.800 \pm 0.030$ & $0.269 \pm 0.020$ & $0.570 \pm 0.100$ & 0.021 \\
\hline & $p+\bar{p}$ & $0.180 \pm 0.030$ & $0.548 \pm 0.010$ & $0.360 \pm 0.020$ & $0.409 \pm 0.010$ & $0.689 \pm 0.030$ & $0.276 \pm 0.019$ & $0.247 \pm 0.040$ & 0.009 \\
\hline
\end{tabular}

